\title{
Scalar Wave Equation Modeling with Time-Space Domain Dispersion-Relation-Based Staggered-Grid Finite-Difference Schemes
}

\author{
by Yang Liu and Mrinal K. Sen
}

\begin{abstract}
The staggered-grid finite-difference (SFD) method is widely used in numerical modeling of wave equations. Conventional SFD stencils for spatial derivatives are usually designed in the space domain. However, when they are used to solve wave equations, it becomes difficult to satisfy the dispersion relations exactly. Liu and Sen (2009c) proposed a new SFD scheme for one-dimensional (1D) scalar wave equation based on the time-space domain dispersion relation and plane wave theory, which is made to satisfy the exact dispersion relation. This new SFD scheme has greater accuracy and better stability than a conventional scheme under the same discretizations. In this paper, we develop this new SFD scheme further for numerical solution of 2D and 3D scalar wave equations. We demonstrate that the modeling accuracy is second order when the conventional $2 M$-th-order space-domain SFD and the second order time-domain finite-difference stencils are directly used to solve the scalar wave equation. However, under the same discretization, our 1D scheme can reach $2 M$-th-order accuracy and is always stable; 2D and 3D schemes can reach $2 M$-th-order accuracy along 8 and 48 directions, respectively, and have better stability. The advantages of the new schemes are also demonstrated with dispersion analysis, stability analysis, and numerical modeling.
\end{abstract}

\section{Introduction}

Numerical solution of seismic wave equation has played an important role in both theoretical and applied seismology. Finite-difference methods (FDMs; e.g., Kelly et al., 1976; Dablain, 1986; Aoi and Fujiwara, 1999; Vossen et al., 2002; Etgen and O'Brien, 2007), finite-element methods (FEMs; e.g., Komatitsch and Vilotte, 1998; Rivière and Wheeler, 2003; Käser and Dumbser, 2006; De Basabe and Sen, 2007, 2009; De Basabe et al., 2008), and pseudospectral methods (PSMs; e.g., Kosloff and Baysal, 1982; Reshef et al., 1988a, 1988b; Liu and Li, 2000; Liu and Wei, 2005) are the three main numerical approaches. The FEMs are generally not widely used because of large memory and computation time requirements, despite the fact that they can handle complicated geometries and boundaries more easily than the FDMs. The PSMs, which are more accurate than the FDMs, are also not very popular for their higher computational cost. Because of their straightforward implementation, requiring small memory and computation time, the FDMs are the most popular methods for seismic modeling (e.g., Virieux, 1986; Yomogida and Etgen, 1993; Igel et al., 1995; Geller and Takeuchi, 1998; Pitarka, 1999; Takeuchi and Geller, 2000; Rojas et al., 2008), migration (e.g., Claerbout, 1985; Ristow and Ruhl, 1994; Zhang et al., 2000) and inversion (e.g., Pratt et al., 1998; Ravaut et al., 2004; Abokhodair, 2009).
To improve the accuracy of FDMs for seismic wave propagation numerical modeling, many variants of the methods have been advanced-these include difference schemes of staggered grid (Virieux, 1984, 1986; Kindelan et al., 1990), variable grid (Wang and Schuster, 1996; Hayashi and Burns, 1999), irregular grid (Opršal and Zahradník, 1999), variable time step (Tessmer, 2000), high-order accuracy (Dablain, 1986; Fornberg, 1987; Crase, 1990; Liu and Wei, 2008; Liu and Sen, 2009d), and implicit formulas (e.g., Emerman et al., 1982; Kosloff et al., 2008; Liu and Sen, 2009a, 2009b).

The staggered-grid finite-difference methods (SFDMs) have greater accuracy and better stability than the conventional-grid FDMs and therefore have been widely used in numerical modeling of acoustic waves (e.g., Etgen and O'Brien, 2007), elastic waves (e.g., Graves, 1996; Moczo et al., 2000, 2002; Mittet, 2002), and viscoacoustic and viscoelastic waves (Robertsson et al., 1994; Bohlen, 2002). Staggered-grid finite-difference (SFD) modeling can also be performed with models that include surface topography (e.g., Robertsson, 1996; Ohminato and Chouet, 1997; Hestholm and Ruud, 1998; Hayashi et al., 2001; Hestholm, 2003; Lombard et al., 2008). Saenger et al., (2000) derived a rotated staggered-grid scheme in which all the medium 
parameters are defined at appropriate positions within an elementary cell for the essential operations. Using this modified grid, it is possible to simulate the propagation of elastic waves in a medium containing cracks, pores, or free surfaces (Saenger and Shapiro, 2002), anisotropy (Saenger and Bohlen, 2004; Bansal and Sen, 2008), and scattering and diffraction by a single crack (Krüger et al., 2005).

It is generally known that the SFD stencils for the spatial derivatives are designed only in the space domain. When these stencils are directly used to solve the wave equations, the dispersion always exists. Finkelstein and Kastner (2007) proposed a new method to derive the finite-difference (FD) coefficients in the joint time-space domain using standard grids. The key idea of this method is that the dispersion relation is completely satisfied at designated frequencies; thus several equations are formed, and the FD coefficients are obtained by solving these equations. This method was developed further for the one-dimensional 1D lossless and boundless wave equation, and its spatial FD coefficients were determined at one designated frequency to obtain arbitraryorder accuracy (Finkelstein and Kastner, 2008). Liu and Sen (2009c) employed a plane wave theory and the Taylor series expansion of dispersion relation to derive the FD coefficients in the joint time-space domain for the scalar wave equation with second-order spatial derivatives. They demonstrated that the method has greater accuracy and better stability than the conventional method. Liu and Sen (2010) designed a spatial FD stencil based on a time-space domain dispersion relation to simulate wave propagation in an acoustic vertically transversely isotropic medium. Two-dimensional dispersion analysis and numerical modeling demonstrated that this stencil has greater precision than one used in a conventional FD.

In this paper, based on the dispersion relation of the FD formula in the time-space domain and the plane wave theory, we derive new spatial SFD coefficients for 2D and 3D scalar wave equation modeling. The coefficients are related to space point number and the Courant number, which are determined by time step, grid size, and velocity. When $2 M$ points are involved in the spatial derivatives and 3 points in the temporal derivatives, the accuracy can be improved from second order of the conventional method to $2 M$ th order of the new method for 1D scalar wave equation modeling (Liu and Sen, 2009c), and 2M-th-order accuracy can be reached along 8 directions for 2D modeling and 48 directions for 3D modeling. Moreover, the new method can adopt a larger Courant number than the conventional method. The advantages of the new method are demonstrated by dispersion analysis, stability analysis, and numerical modeling.

This paper mainly includes six sections. First, we prove that the accuracy of scalar wave equation modeling using the conventional SFD operators is of the second order. Second, we derive new SFD coefficients for 2D and 3D scalar wave equation modeling. Third and fourth, dispersion and stability analyses are carried out for both the conventional and the new methods. Fifth, the conventional and the new methods are used to perform numerical modeling, both in homogeneous and inhomogeneous media. Finally, we draw conclusions.

\section{Accuracy of Scalar Wave Equation Modeling by the Conventional SFDM}

We start with the 1D scalar wave equation in inhomogeneous media (Claerbout, 1985)

$$
\frac{\partial}{\partial x}\left(\frac{1}{\rho} \frac{\partial p}{\partial x}\right)=\frac{1}{K} \frac{\partial^{2} p}{\partial t^{2}}
$$

where $\rho$ is the density; $K$ is the bulk modulus, $K=$ $\lambda+2 \mu=\rho v^{2}$ in which $v$ is the velocity; and $p$ represents the pressure.

The following second-order FD is usually used:

$$
\frac{\partial^{2} p}{\partial t^{2}} \approx \frac{\delta^{2} p}{\delta t^{2}}=\frac{1}{\tau^{2}}\left[-2 p_{0}^{0}+\left(p_{0}^{-1}+p_{0}^{1}\right)\right]
$$

where

$$
p_{m}^{n}=p(x+m h, t+n \tau),
$$

$h$ is the grid size, $x$ is the space coordinate, $t$ is time, and $\tau$ is the time step. Generally, the modeling accuracy is improved by high-order FD for the spatial derivatives; the $2 M$-th-order SFD formula for the first-order derivatives is (Kindelan et al., 1990)

$$
\frac{\partial p}{\partial x}=\frac{1}{h} \sum_{m=1}^{M} a_{m}\left(p_{m-1 / 2}^{0}-p_{-m+1 / 2}^{0}\right) .
$$

Assuming the media are homogeneous and substituting equation (2) and equation (4) into equation (1), we have

$$
\begin{aligned}
& \frac{1}{h^{2}} \sum_{m=1}^{M} \sum_{n=1}^{M} a_{m} a_{n}\left[\left(p_{m+n-1}^{0}-p_{m-n}^{0}\right)-\left(p_{-m+n}^{0}-p_{-m-n+1}^{0}\right)\right] \\
& \quad \approx \frac{1}{v^{2} \tau^{2}}\left(p_{0}^{1}+p_{0}^{-1}-2 p_{0}^{0}\right)
\end{aligned}
$$

In the conventional method, FD coefficients for the spatial derivatives are determined in the space domain. Using the plane wave theory, we let

$$
p_{m}^{n}=e^{i[k(x+m h)-\omega(t+n \tau)]}=e^{i(k x-\omega t)} e^{i(m k h-n \omega \tau)},
$$

where, $k$ is the wavenumber, $\omega$ is the angular frequency, and $i=\sqrt{-1}$. Substituting equation (6) into equation (4) and simplifying it, we have

$$
k \approx \frac{2}{h} \sum_{m=1}^{M} a_{m} \sin ((m-0.5) k h) .
$$

Using the Taylor series expansion, we obtain

$$
k \approx \frac{2}{h} \sum_{m=1}^{M} a_{m} \sum_{j=1}^{\infty} \frac{(-1)^{j-1}((m-0.5) h k)^{2 j-1}}{(2 j-1) !} .
$$

By comparing the coefficients of $k, k^{3}, \ldots, k^{2 M-1}, M$ equations can be obtained to solve SFD coefficients $a_{1}, a_{2}, \ldots ., a_{M}$ (Kindelan et al., 1990; Pei, 2004; Liu and 
Sen, 2009b). Then, the error of SFD on spatial derivatives is derived from equation (8) as follows:

$$
\frac{2}{h} \sum_{m=1}^{M} a_{m} \sum_{j=M+1}^{\infty} \frac{(-1)^{j-1}((m-0.5) h k)^{2 j-1}}{(2 j-1) !} .
$$

Therefore, we obtain

$$
\begin{aligned}
& \frac{2}{h} \sum_{m=1}^{M} a_{m} \sin ((m-0.5) k h) \\
& \quad=k+\frac{2}{h} \sum_{j=M+1}^{\infty}\left(\sum_{m=1}^{M}(m-0.5)^{2 j-1} a_{m}\right) \frac{(-1)^{j-1}(h k)^{2 j-1}}{(2 j-1) !} .
\end{aligned}
$$

Substituting equation (6) into equation (2) and using the Taylor series expansion, we obtain

$$
-\omega^{2} \approx \frac{1}{\tau^{2}}[-2+2 \cos (\omega \tau)]=-\omega^{2}+\frac{2}{\tau^{2}} \sum_{j=2}^{\infty} \frac{(-1)^{j}(\omega \tau)^{2 j}}{(2 j) !} .
$$

When the 1D space domain SFD and time domain FD formulas are directly used to solve the 1D scalar wave equation, the error function is expressed as equation (A2) (see Appendix A). Because the minimum power of $h$ in the error function (A2) is 2, FD accuracy is second order. Therefore, when the $2 M$-th-order space domain SFD and the second-order time domain FD stencils are used to solve the 1D scalar wave equation, the accuracy is second order. The conclusion is obviously the same for the 2D and 3D scalar wave equations. Note that increasing $M$ may decrease the magnitude of FD error without increasing the accuracy order. The main reason is that the wave equation is solved in both the spatial and the temporal domains simultaneously, but SFD stencils are designed in either the spatial or the temporal domain, but not both.

\section{Time-Space Domain Dispersion-Relation-Based Spatial SFD Stencils}

In this section, we derive new spatial SFD coefficients for 2D and 3D scalar wave modeling based on the time-space domain dispersion relations. We start with a 1D case.

\section{D SFD Stencil}

Substituting equation (6) into equation (5) and simplifying it, we obtain

$$
-\frac{4}{h^{2}}\left[\sum_{m=1}^{M} a_{m} \sin ((m-0.5) k h)\right]^{2} \approx-\frac{4}{v^{2} \tau^{2}}[\sin (0.5 \omega \tau)]^{2} .
$$

Using the Taylor series expansion for sine functions, $r=v \tau / h$ and $\omega=v k$, we obtain SFD coefficients for 1D scalar wave equation modeling (Liu and Sen, 2009c):

$$
a_{m}=\frac{(-1)^{m+1}}{2 m-1} \prod_{1 \leq n \leq M, n \neq m}\left|\frac{(2 n-1)^{2}-r^{2}}{(2 n-1)^{2}-(2 m-1)^{2}}\right|,
$$

where $r \leq 1$. The SFD modeling has $2 M$-th-order accuracy. When $r=0$, the SFD coefficients are the same as those from the conventional method (Kindelan et al., 1990; Pei, 2004; Liu and Sen, 2009b).

\section{D SFD Stencil}

The 2D scalar wave equation is (Claerbout, 1985)

$$
\frac{\partial}{\partial x}\left(\frac{1}{\rho} \frac{\partial P}{\partial x}\right)+\frac{\partial}{\partial z}\left(\frac{1}{\rho} \frac{\partial P}{\partial z}\right)=\frac{1}{K} \frac{\partial^{2} P}{\partial t^{2}} .
$$

Because the same SFD is usually used for spatial derivatives, we let

$$
\frac{\partial p}{\partial x} \approx \frac{\delta p}{\delta x}=\frac{1}{h} \sum_{m=1}^{M}\left(p_{m-1 / 2,0}^{0}-p_{-m+1 / 2,0}^{0}\right),
$$

and

$$
\frac{\partial p}{\partial z} \approx \frac{\delta p}{\delta z}=\frac{1}{h} \sum_{m=1}^{M}\left(p_{0, m-1 / 2}^{0}-p_{0,-m+1 / 2}^{0}\right),
$$

where

$$
p_{m, j}^{n}=p(x+m h, z+j h, t+n \tau) .
$$

The second-order FD stencil for the temporal derivative is

$$
\frac{\partial^{2} p}{\partial t^{2}} \approx \frac{\delta^{2} p}{\delta t^{2}}=\frac{1}{\tau^{2}}\left[-2 p_{0,0}^{0}+\left(p_{0,0}^{-1}+p_{0,0}^{1}\right)\right]
$$

Using equation (15a), equation (15b), and equation (17), equation (14) is changed as follows:

$$
\begin{aligned}
& \frac{1}{h^{2}} \sum_{m=1}^{M} \sum_{n=1}^{M} a_{m} a_{n}\left[\left(p_{m+n-1,0}^{0}-p_{m-n, 0}^{0}\right)\right. \\
& \left.\quad-\left(p_{-m+n, 0}^{0}-p_{-m-n+1,0}^{0}\right)\right] \\
& \quad+\frac{1}{h^{2}} \sum_{m=1}^{M} \sum_{n=1}^{M} a_{m} a_{n}\left[\left(p_{0, m+n-1}^{0}-p_{0, m-n}^{0}\right)\right. \\
& \left.\quad-\left(p_{0,-m+n}^{0}-p_{0,-m-n+1}^{0}\right)\right] \\
& \approx \frac{1}{v^{2} \tau^{2}}\left(p_{0,0}^{1}+p_{0,0}^{-1}-2 p_{0,0}^{0}\right)
\end{aligned}
$$

Let

$$
p_{m, j}^{n}=e^{i\left[k_{x}(x+m h)+k_{z}(z+j h)-\omega(t+n \tau)\right]} .
$$

Substituting equation (19) into equation (18) and simplifying it, we obtain

$$
\begin{aligned}
& {\left[\sum_{m=1}^{M} a_{m} \sin \left((m-0.5) k_{x} h\right)\right]^{2}} \\
& \quad+\left[\sum_{m=1}^{M} a_{m} \sin \left((m-0.5) k_{z} h\right)\right]^{2} \\
& \approx\left[r^{-1} \sin (0.5 \omega \tau)\right]^{2} .
\end{aligned}
$$


Let

$$
k_{x}=k \cos \theta \quad \text { and } \quad k_{z}=k \sin \theta,
$$

where $\theta$ is a propagation direction angle of the plane wave. Then equation (20) changes to

$$
\begin{aligned}
& {\left[\sum_{m=1}^{M} a_{m} \sin ((m-0.5) k h \cos \theta)\right]^{2}} \\
& \quad+\left[\sum_{m=1}^{M} a_{m} \sin ((m-0.5) k h \sin \theta)\right]^{2} \\
& \approx\left[r^{-1} \sin (0.5 \omega \tau)\right]^{2} .
\end{aligned}
$$

Using the Taylor series expansion for sine functions, $r=v \tau / h$ and $\omega=v k$, we have

$$
\begin{aligned}
& {\left[\sum_{n=1}^{\infty} \sum_{m=1}^{M}((2 m-1) \cos \theta)^{2 n-1} a_{m} \frac{(-1)^{n-1}(0.5 k h)^{2 n-1}}{(2 n-1) !}\right]^{2}} \\
& \quad+\left[\sum_{n=1}^{\infty} \sum_{m=1}^{M}((2 m-1) \sin \theta)^{2 n-1} a_{m} \frac{(-1)^{n-1}(0.5 k h)^{2 n-1}}{(2 n-1) !}\right]^{2} \\
& \quad \approx\left[\sum_{n=1}^{\infty} r^{2 n-2} \frac{(-1)^{n-1}(0.5 k h)^{2 n-1}}{(2 n-1) !}\right]^{2} .
\end{aligned}
$$

Here, let

$$
\begin{aligned}
& {\left[\sum_{n=1}^{\infty} b_{n} c_{n}(0.5 k h)^{2 n-1}\right]^{2}+\left[\sum_{n=1}^{\infty} b_{n} d_{n}(0.5 k h)^{2 n-1}\right]^{2}} \\
& \quad \approx\left[\sum_{n=1}^{\infty} r^{2 n-2} \beta_{n}(0.5 k h)^{2 n-1}\right]^{2} .
\end{aligned}
$$

Comparing coefficients of $k^{2 n}$, we obtain

$$
b_{1}^{2}\left(c_{1}^{2}+d_{1}^{2}\right)=1 \quad(n=1),
$$

and

$$
\begin{aligned}
& \sum_{j=1}^{n} b_{j} b_{n+1-j}\left(c_{j} c_{n+1-j}+d_{j} d_{n+1-j}\right) \\
& \quad=\sum_{j=1}^{n} \beta_{j} \beta_{n+1-j} r^{2 n-2} \\
& (n=2,3, \ldots, M) .
\end{aligned}
$$

Equation (26a) gives $b_{1}= \pm 1$. When $b_{1}$ changes from 1 to $-1, a_{m}$ will change to $-a_{m}$, which does not affect the final results. Therefore, we let

$$
b_{1}=1 .
$$

Then, equation (26b) can be rewritten as follows

$$
b_{n}=\frac{\left(\sum_{j=1}^{n} \beta_{j} \beta_{n+1-j}\right) r^{2 n-2}-\sum_{j=2}^{n-1}\left[b_{j} b_{n+1-j}\left(c_{j} c_{n+1-j}+d_{j} d_{n+1-j}\right)\right]}{2\left(c_{1} c_{n}+d_{1} d_{n}\right)} \quad(n=2,3, \ldots, M) .
$$

$$
\beta_{n}=\frac{(-1)^{n-1}}{(2 n-1) !},
$$

$$
b_{n}=\sum_{m=1}^{M}(2 m-1)^{2 n-1} a_{m},
$$

$$
c_{n}=(\cos \theta)^{2 n-1} \beta_{n},
$$

and

$$
d_{n}=(\sin \theta)^{2 n-1} \beta_{n}
$$

Because $\beta_{n}, c_{n}$, and $d_{n}$ are known for the given $\theta$, using equation (27), $b_{2}$ can be obtained from equation (28); using the known $b_{1}$ and $b_{2}, b_{3}$ can be obtained from equation (28). Similarly, $b_{4}, \ldots, b_{M}$ can be obtained from equation (28). After $b_{1}, b_{2}, \ldots, b_{M}$ are known, using equation (24b), we have the following matrix equation:

$\left[\begin{array}{cccc}1^{0} & 3^{0} & \cdots & (2 M-1)^{0} \\ 1^{2} & 3^{2} & \cdots & (2 M-1)^{2} \\ \vdots & \vdots & \vdots & \vdots \\ 1^{2 M-2} & 3^{2 M-2} & \cdots & (2 M-1)^{2 M-2}\end{array}\right]\left[\begin{array}{c}1 a_{1} \\ 3 a_{2} \\ \vdots \\ (2 M-1) a_{M}\end{array}\right]$

$$
=\left[\begin{array}{c}
1 \\
b_{2} \\
\vdots \\
b_{M}
\end{array}\right] \text {. }
$$

then 
The coefficients $a_{m}(m=1,2, \ldots, M)$ are obtained by solving these equations. Some special cases are given in Appendix B.

Notice that $b_{2}, b_{3}, \ldots, b_{M}$ are the function of $\theta$, as are $a_{m}$. To obtain a single set of coefficients, we may choose an optimal angle. From equations (24a-d) and (28), we can see that when $\theta$ is changed to $n \pi / 2 \pm \theta, b_{2}, b_{3}, \ldots, b_{M}$ are changeless, as are $a_{m}$. That is,

$$
a_{m}(\theta)=a_{m}(n \pi / 2 \pm \theta) .
$$

Therefore,

$$
a_{m}(\pi / 8)=a_{m}(\pi / 8 \pm n \pi / 4) .
$$

If $\theta=\pi / 8$ is used to solve the equation (28) and equation (29), SFD modeling can reach the highest $2 M$-th-order accuracy along these eight directions: $\theta=(2 n-1) \pi / 8, \quad(n=$ $1,2, \ldots, 8)$. Therefore, we calculate $a_{m}$ by using $\theta=\pi / 8$.

\section{D SFD Stencil}

For the 3D scalar wave equation,

$$
\frac{\partial}{\partial x}\left(\frac{1}{\rho} \frac{\partial P}{\partial x}\right)+\frac{\partial}{\partial y}\left(\frac{1}{\rho} \frac{\partial P}{\partial y}\right)+\frac{\partial}{\partial z}\left(\frac{1}{\rho} \frac{\partial P}{\partial z}\right)=\frac{1}{K} \frac{\partial^{2} P}{\partial t^{2}},
$$

Comparing coefficients of $k^{2 n}$ in equation (33), we obtain

$$
b_{1}^{2}\left(f_{1}^{2}+g_{1}^{2}+d_{1}^{2}\right)=1 \quad(n=1),
$$

and

$$
\begin{aligned}
& \sum_{j=1}^{n} b_{j} b_{n+1-j}\left(f_{j} f_{n+1-j}+g_{j} g_{n+1-j}+d_{j} d_{n+1-j}\right) \\
& \quad=\sum_{j=1}^{n} \beta_{j} \beta_{n+1-j} r^{2 n-2} \\
& (n=2,3, \ldots, M) .
\end{aligned}
$$

Equation (35a) gives $b_{1}= \pm 1$. When $b_{1}$ changes from 1 to $-1, a_{m}$ will change to $-a_{m}$, which does not affect the final results. Therefore, we let

$$
b_{1}=1 \text {. }
$$

Then, equation (35b) can be rewritten as

$$
b_{n}=\frac{\left(\sum_{j=1}^{n} \beta_{j} \beta_{n+1-j}\right) r^{2 n-2}-\sum_{j=2}^{n-1}\left[b_{j} b_{n+1-j}\left(f_{j} f_{n+1-j}+g_{j} g_{n+1-j}+d_{j} d_{n+1-j}\right)\right]}{2\left(f_{1} f_{n}+g_{1} g_{n}+d_{1} d_{n}\right)} \quad(n=2,3, \ldots, M) .
$$

we can obtain the following equation, which is similar to equation (25):

$$
\begin{aligned}
& {\left[\sum_{n=1}^{\infty} b_{n} f_{n}(0.5 k h)^{2 n-1}\right]^{2}+\left[\sum_{n=1}^{\infty} b_{n} g_{n}(0.5 k h)^{2 n-1}\right]^{2}} \\
& \quad+\left[\sum_{n=1}^{\infty} b_{n} d_{n}(0.5 k h)^{2 n-1}\right]^{2} \\
& \approx\left[\sum_{n=1}^{\infty} r^{2 n-2} \beta_{n}(0.5 k h)^{2 n-1}\right]^{2},
\end{aligned}
$$

where

$$
f_{n}=(\cos \theta \cos \phi)^{2 n-1} \beta_{n}, \quad g_{n}=(\cos \theta \sin \phi)^{2 n-1} \beta_{n},
$$

$\theta$ is the plane wave propagation angle measured from the horizontal plane perpendicular to $z$ axis, and $\phi$ is the azimuth of the plane wave.
Because $\beta_{n}, f_{n}, g_{n}$, and $d_{n}$ are known for the given $\theta$ and $\phi$, using the known $b_{1}$ and equation (37), $b_{2}, b_{3}, \ldots, b_{M}$ can be obtained. The coefficients $a_{m}$ are obtained by solving equation (29). It is obvious that if $\theta=0$ and $\phi=\pi / 8$ are used in equation (37), the SFD modeling can reach the highest $2 M$ th-order accuracy along 48 directions: $\theta=(m-1) \pi$, $\phi=(2 n-1) \pi / 8, \quad(m=1, \quad 2 ; \quad n=1,2, \ldots, 8) ; \quad \theta=$ $(2 m-1) \pi / 8, \phi=(n-1) \pi / 2(n=1,2, \ldots, 8 ; m=1,2$, 3, 4. Therefore, we calculate $a_{m}$ by using $\theta=0$ and $\phi=\pi / 8$. It can be deduced that 3D SFD coefficients from these two angles are the same as 2D SFD coefficients from angle $\pi / 8$. Table 1 lists SFD coefficients $a_{m}$ of the old and new methods for $M=20$ and different values of $r$.

\section{Dispersion Analysis}

\section{D Dispersion Analysis}

We use equation (12) to define a parameter $\delta$ to describe 1D dispersion of FD: 
Table 1

SFD Coefficients $a_{m}$ of Old and New Methods for $M=20$ and Different $r$

\begin{tabular}{|c|c|c|c|c|c|}
\hline \multirow[b]{2}{*}{$m$} & \multirow[b]{2}{*}{ Old Method } & \multicolumn{2}{|c|}{$r=0.2$} & \multicolumn{2}{|c|}{$r=0.3$} \\
\hline & & New 1D Method & New 2D/3D Method & New 1D Method & New 2D/3D Method \\
\hline 1 & $0.12574 \times 10^{1}$ & $0.12463 \times 10^{1}$ & $0.12426 \times 10^{1}$ & $0.12326 \times 10^{1}$ & $0.12243 \times 10^{1}$ \\
\hline 2 & $-0.12641 \times 10^{0}$ & $-0.12082 \times 10^{0}$ & $-0.11895 \times 10^{0}$ & $-0.11390 \times 10^{0}$ & $-0.10972 \times 10^{0}$ \\
\hline 3 & $0.37233 \times 10^{-1}$ & $0.35485 \times 10^{-1}$ & $0.34902 \times 10^{-1}$ & $0.33332 \times 10^{-1}$ & $0.32030 \times 10^{-1}$ \\
\hline 4 & $-0.14041 \times 10^{-1}$ & $-0.13371 \times 10^{-1}$ & $-0.13148 \times 10^{-1}$ & $-0.12548 \times 10^{-1}$ & $-0.12049 \times 10^{-1}$ \\
\hline 5 & $0.56625 \times 10^{-2}$ & $0.53907 \times 10^{-2}$ & $0.53001 \times 10^{-2}$ & $0.50567 \times 10^{-2}$ & $0.48543 \times 10^{-2}$ \\
\hline 6 & $-0.22744 \times 10^{-2}$ & $-0.21649 \times 10^{-2}$ & $-0.21283 \times 10^{-2}$ & $-0.20303 \times 10^{-2}$ & $-0.19487 \times 10^{-2}$ \\
\hline 7 & $0.87683 \times 10^{-3}$ & $0.83453 \times 10^{-3}$ & $0.82042 \times 10^{-3}$ & $0.78256 \times 10^{-3}$ & $0.75107 \times 10^{-3}$ \\
\hline 8 & $-0.31710 \times 10^{-3}$ & $-0.30179 \times 10^{-3}$ & $-0.29668 \times 10^{-3}$ & $-0.28297 \times 10^{-3}$ & $-0.27157 \times 10^{-3}$ \\
\hline 9 & $0.10581 \times 10^{-3}$ & $0.10069 \times 10^{-3}$ & $0.98985 \times 10^{-4}$ & $0.94409 \times 10^{-4}$ & $0.90602 \times 10^{-4}$ \\
\hline 10 & $-0.32129 \times 10^{-4}$ & $-0.30575 \times 10^{-4}$ & $-0.30056 \times 10^{-4}$ & $-0.28666 \times 10^{-4}$ & $-0.27510 \times 10^{-4}$ \\
\hline 11 & $0.87668 \times 10^{-5}$ & $0.83426 \times 10^{-5}$ & $0.82011 \times 10^{-5}$ & $0.78216 \times 10^{-5}$ & $0.75059 \times 10^{-5}$ \\
\hline 12 & $-0.21218 \times 10^{-5}$ & $-0.20191 \times 10^{-5}$ & $-0.19849 \times 10^{-5}$ & $-0.18930 \times 10^{-5}$ & $-0.18165 \times 10^{-5}$ \\
\hline 13 & $0.44897 \times 10^{-6}$ & $0.42724 \times 10^{-6}$ & $0.41999 \times 10^{-6}$ & $0.40054 \times 10^{-6}$ & $0.38437 \times 10^{-6}$ \\
\hline 14 & $-0.81650 \times 10^{-7}$ & $-0.77697 \times 10^{-7}$ & $-0.76378 \times 10^{-7}$ & $-0.72841 \times 10^{-7}$ & $-0.69899 \times 10^{-7}$ \\
\hline 15 & $0.12490 \times 10^{-7}$ & $0.11885 \times 10^{-7}$ & $0.11683 \times 10^{-7}$ & $0.11142 \times 10^{-7}$ & $0.10692 \times 10^{-7}$ \\
\hline 16 & $-0.15615 \times 10^{-8}$ & $-0.14859 \times 10^{-8}$ & $-0.14606 \times 10^{-8}$ & $-0.13930 \times 10^{-8}$ & $-0.13367 \times 10^{-8}$ \\
\hline 17 & $0.15310 \times 10^{-9}$ & $0.14569 \times 10^{-9}$ & $0.14322 \times 10^{-9}$ & $0.13658 \times 10^{-9}$ & $0.13106 \times 10^{-9}$ \\
\hline 18 & $-0.11036 \times 10^{-10}$ & $-0.10501 \times 10^{-10}$ & $-0.10323 \times 10^{-10}$ & $-0.98446 \times 10^{-11}$ & $-0.94468 \times 10^{-11}$ \\
\hline 19 & $0.51973 \times 10^{-12}$ & $0.49455 \times 10^{-12}$ & $0.48615 \times 10^{-12}$ & $0.46363 \times 10^{-12}$ & $0.44490 \times 10^{-12}$ \\
\hline 20 & $-0.11995 \times 10^{-13}$ & $-0.11414 \times 10^{-13}$ & $-0.11220 \times 10^{-13}$ & $-0.10700 \times 10^{-13}$ & $-0.10267 \times 10^{-13}$ \\
\hline
\end{tabular}

(a)

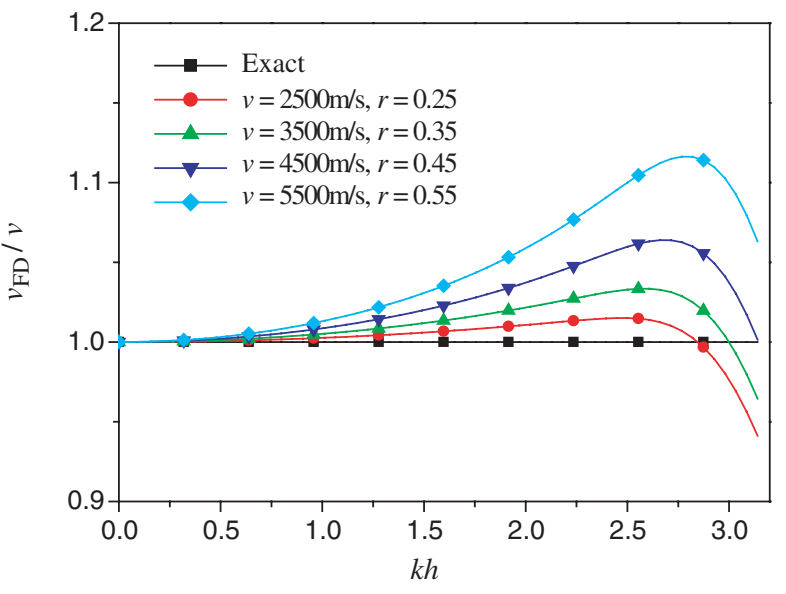

(b)

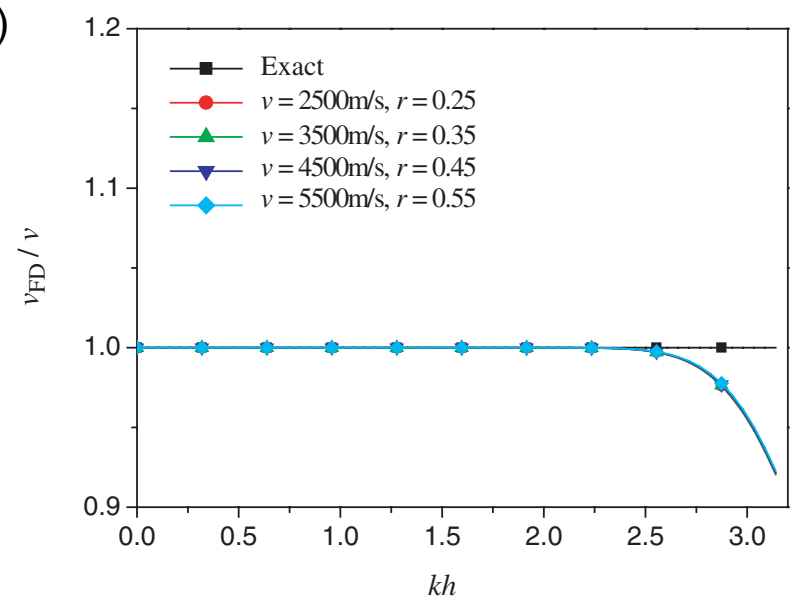

Figure 1. 1D dispersion curves of (a) the conventional and (b) the new methods for different velocities. $\tau=0.001 \mathrm{~s}$, $h=10 \mathrm{~m}, M=20$. The color version of this figure is available only in the electronic edition.

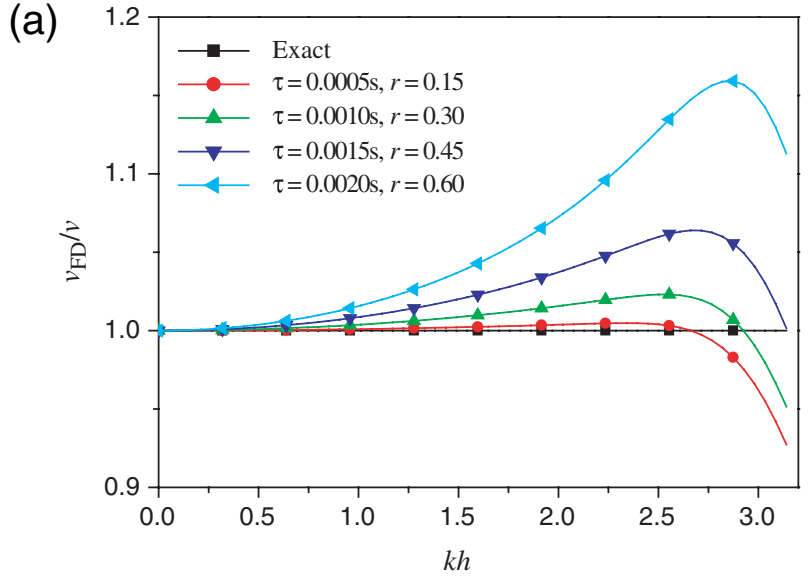

(b)

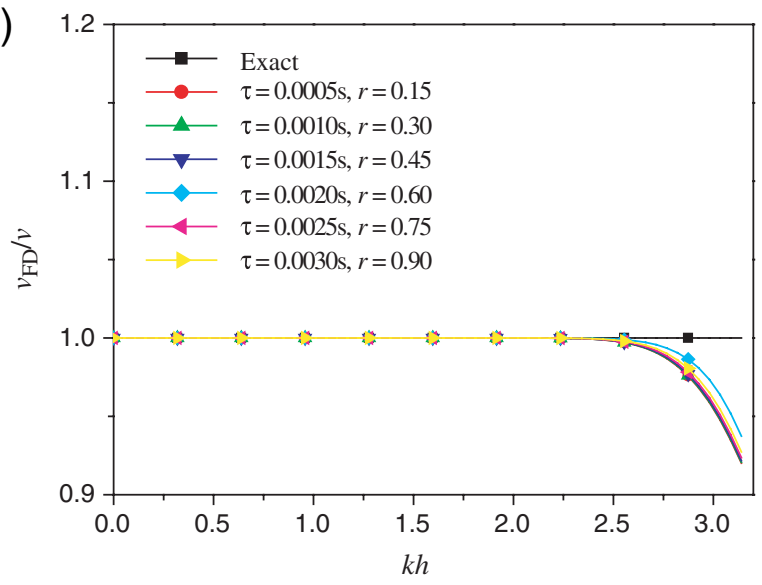

Figure 2. 1D dispersion curves of (a) the conventional and (b) the new methods for different time steps. $\tau=0.0025 \mathrm{~s}$, $0.0030 \mathrm{~s}$; that is, $r=0.75,0.9$ is added for the new method. $v=3000 \mathrm{~m} / \mathrm{s}, h=10 \mathrm{~m}, M=20$. The color version of this figure is available only in the electronic edition. 
(a)

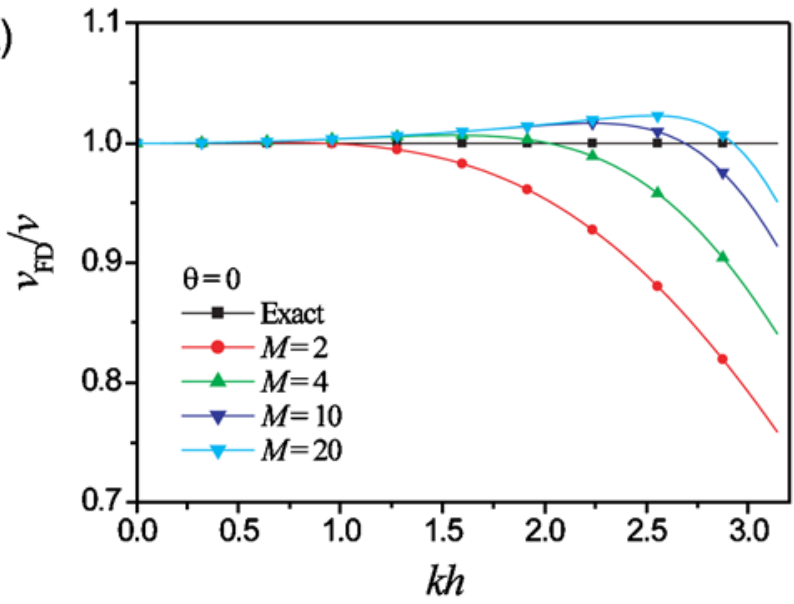

(c)

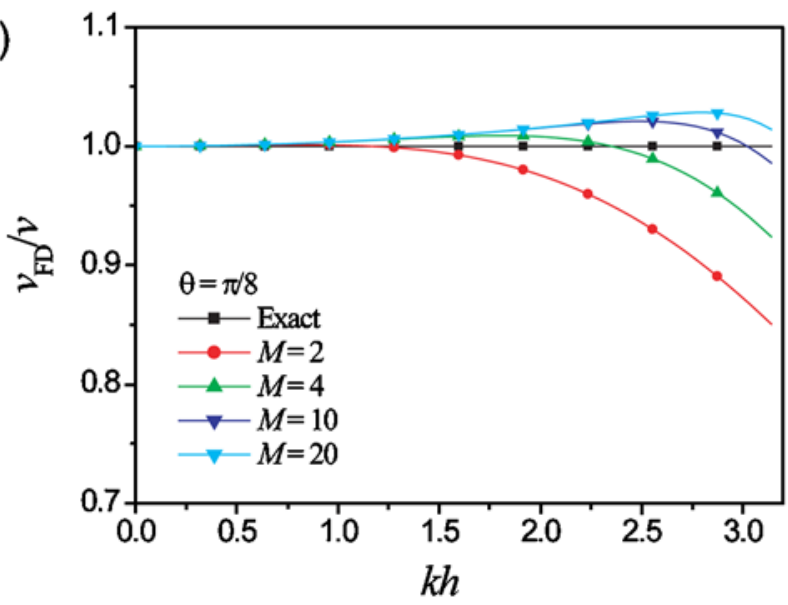

(e)

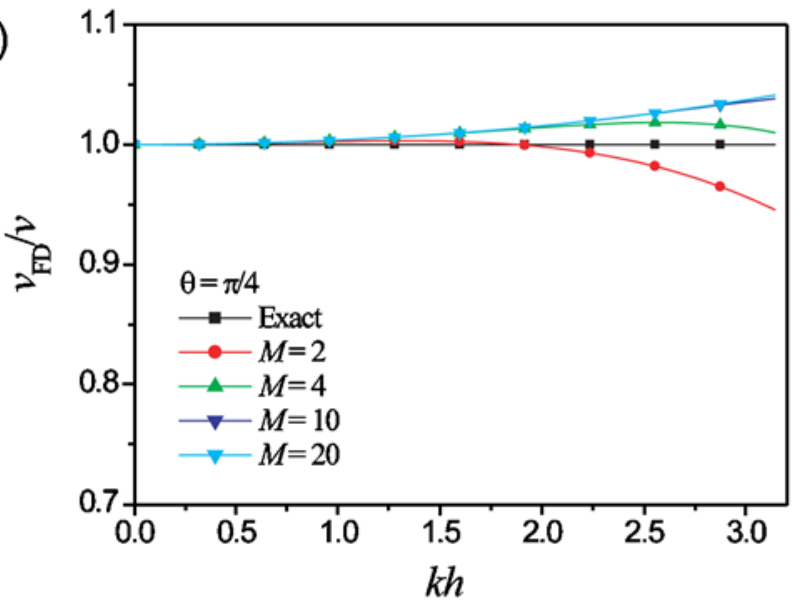

(b)

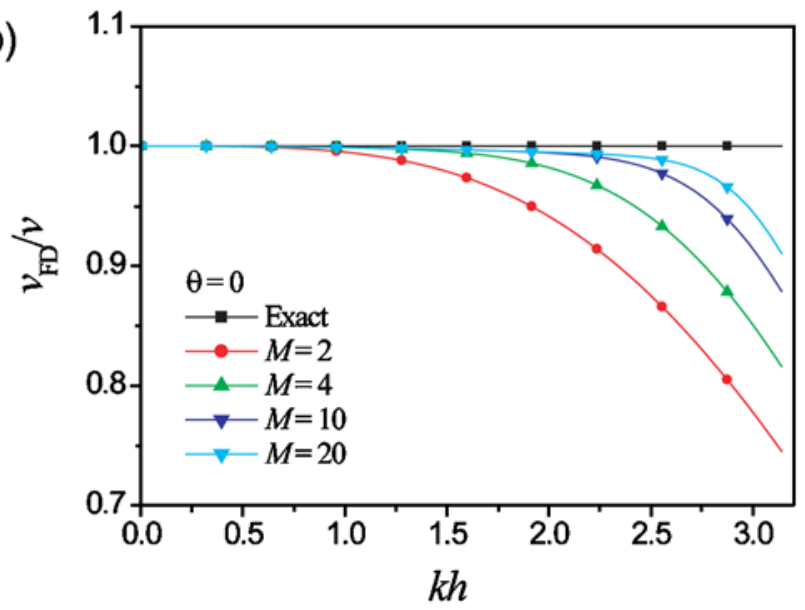

(d)

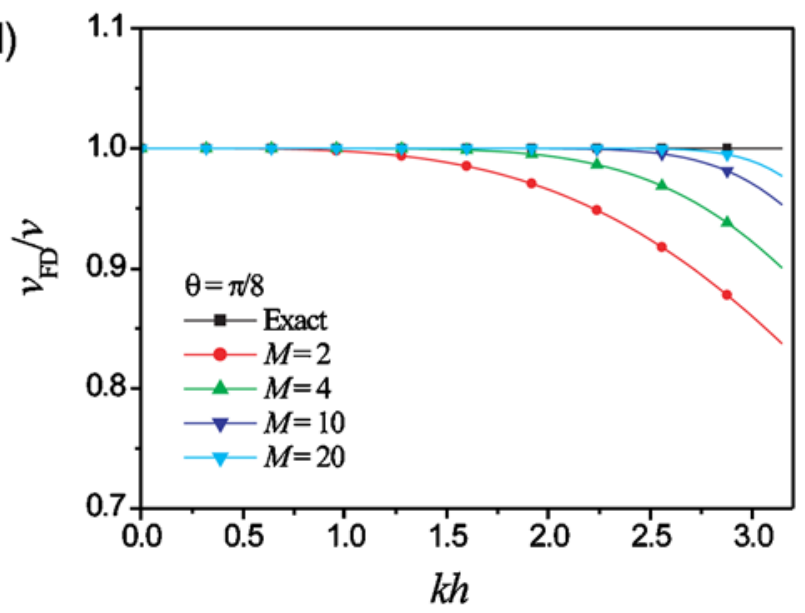

(f)

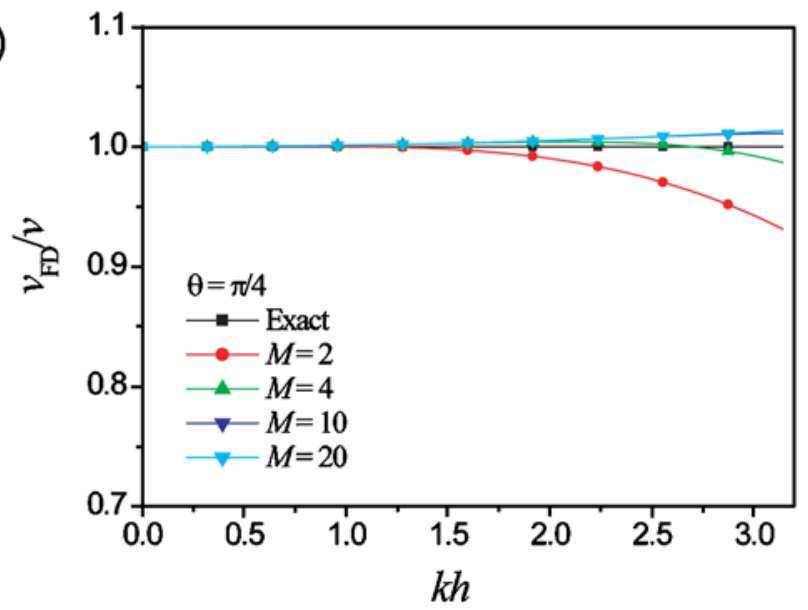

Figure 3. 2D dispersion curves of (a, c, e) the conventional and (b, d, f) the new methods for different propagation angles and different space point numbers $2 M+1 . v=3000 \mathrm{~m} / \mathrm{s}, \tau=0.001 \mathrm{~s}, h=10 \mathrm{~m}$. The color version of this figure is available only in the electronic edition. 
(a)

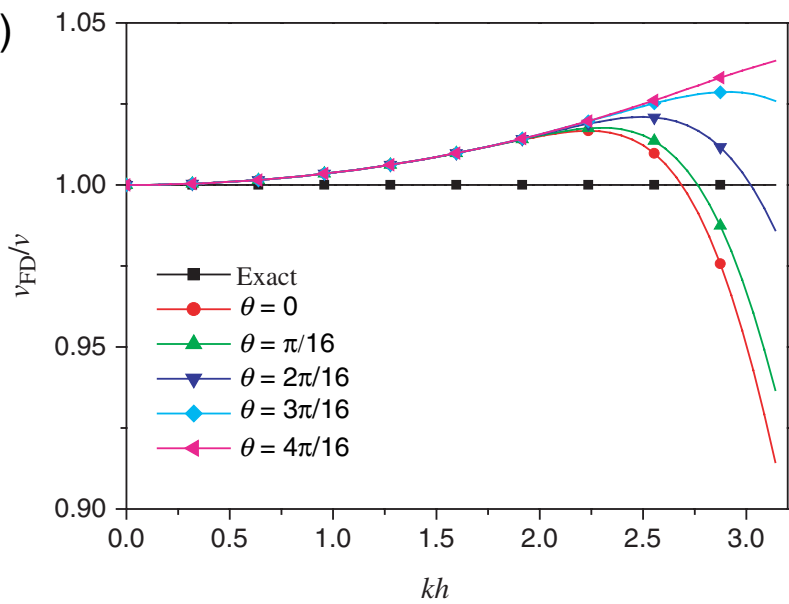

(b)

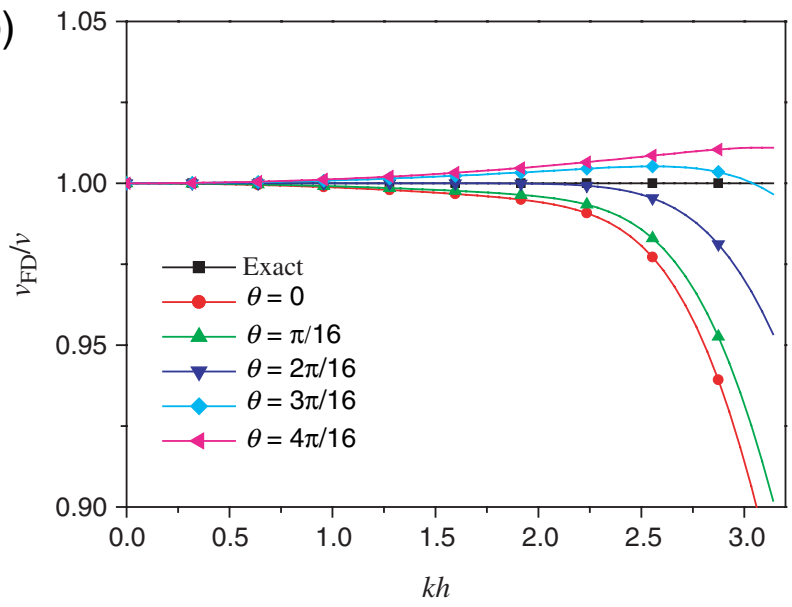

Figure 4. 2D of dispersion curves of (a) the conventional and (b) the new methods. $v=3000 \mathrm{~m} / \mathrm{s}, \tau=0.001 \mathrm{~s}, h=10 \mathrm{~m}$, $M=10$. The color version of this figure is available only in the electronic edition.

$$
\delta=\frac{v_{\mathrm{FD}}}{v}=\frac{2}{r k h} \sin ^{-1}\left(r \sum_{m=1}^{M} a_{m} \sin [(m-0.5) k h]\right) .
$$

If $\delta$ equals 1 , there is no dispersion. If $\delta$ is far from 1 , a large dispersion will occur. Because $k h$ is equal to $\pi$ at the Nyquist frequency, $k h$ only ranges from 0 to $\pi$ when calculating $\delta$.

Next, we compare the conventional and the new methods by the dispersion curves for different velocities and time steps.

Figure 1 illustrates the effect of velocity on dispersion for various parameters From this figure, it follows that the conventional dispersion curves change significantly with the variation of velocity, while the new dispersion curves change a little. The dispersion characteristics of the new method, mainly dependent on $M$, are more accurate and stable than the conventional method.

Figure 2 shows the variation of the dispersion parameter $\delta$ with $k h$ for different time steps. For the conventional method, its dispersion increases with the increase of time step. Furthermore, larger time steps, such as $0.0025 \mathrm{~s}$ and (a)

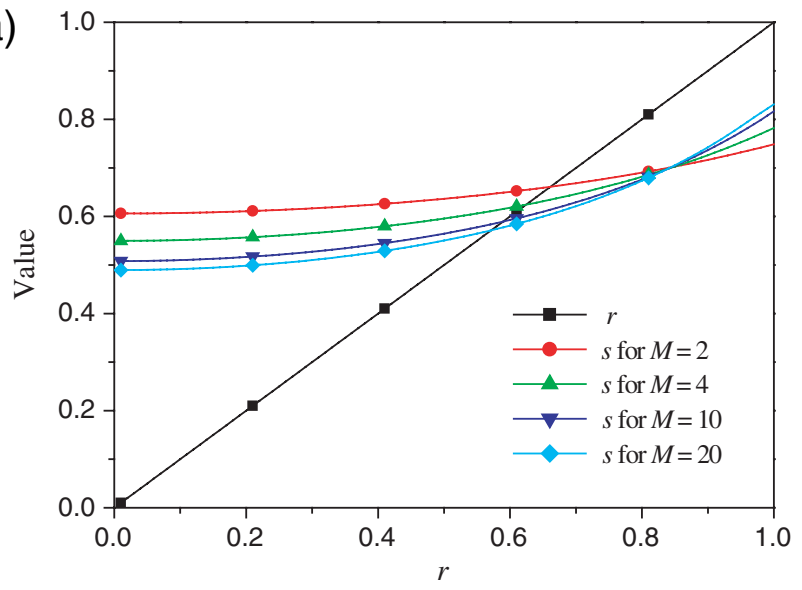

(b)

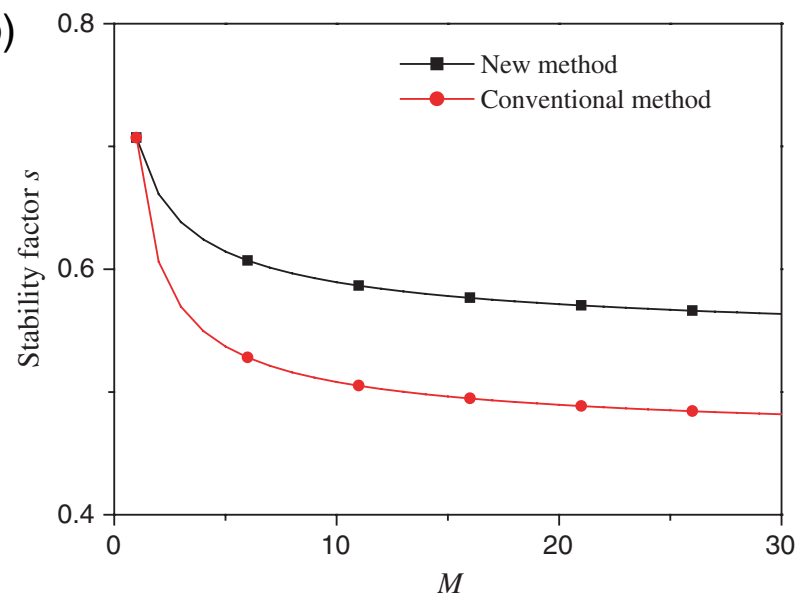

Figure 5. (a) The variation of stability factor $s$ with $M$ and $r$ for the new method. (b) Stability condition of the conventional and the new methods; the stability factor of the new method shown here is the maximum value of $s$ satisfying equation (41). The method is stable when $r \leq s$. The color version of this figure is available only in the electronic edition.

$0.0030 \mathrm{~s}$ in this example, make the conventional recursion unstable. However, the new recursion is still stable for these larger time steps, and its dispersion varies slightly with the variation of time step. Therefore, the new method can adopt larger time steps and attain greater precision.

\section{D Dispersion Analysis}

2D dispersion $\delta(\theta)$ is defined by using equation (20):

$$
\delta(\theta)=\frac{v_{\mathrm{FD}}}{v}=\frac{2}{r k h} \sin ^{-1}\left(r \sqrt{q_{2}}\right),
$$

where

$$
\begin{aligned}
q_{2}= & \left(\sum_{m=1}^{M} a_{m} \sin [(m-0.5) k h \cos \theta]\right)^{2} \\
& +\left(\sum_{m=1}^{M} a_{m} \sin [(m-0.5) k h \sin \theta]\right)^{2} .
\end{aligned}
$$


(a) Trace No. (1) (2) (3) (4) (5) (6)

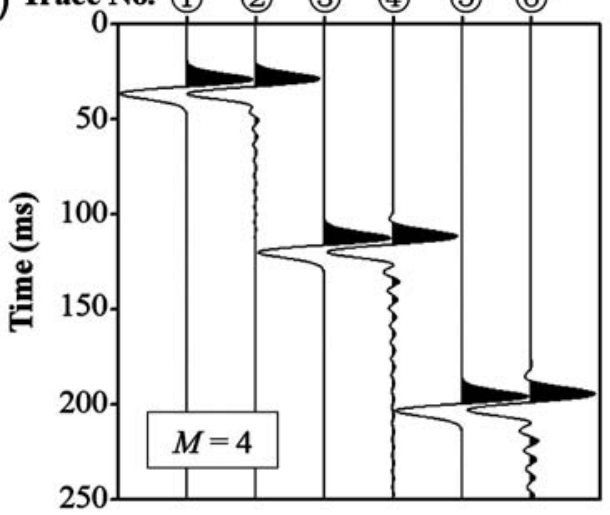

(b) Trace No. (1) (2) (3) (4) (5) (6)

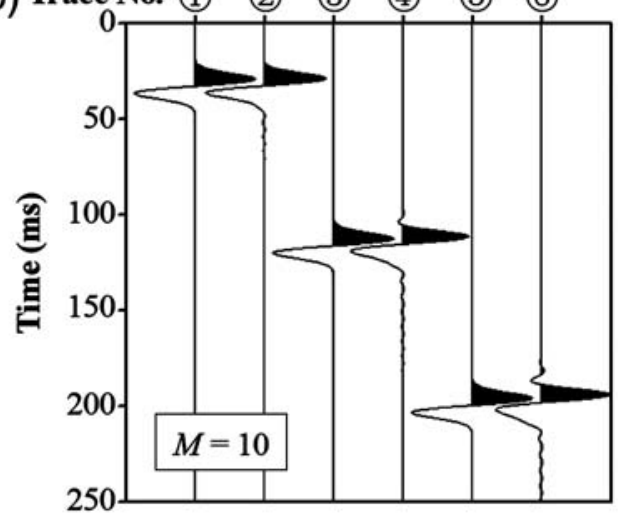

(c) Trace No. (1) (2) (3) (4) (5) (6)

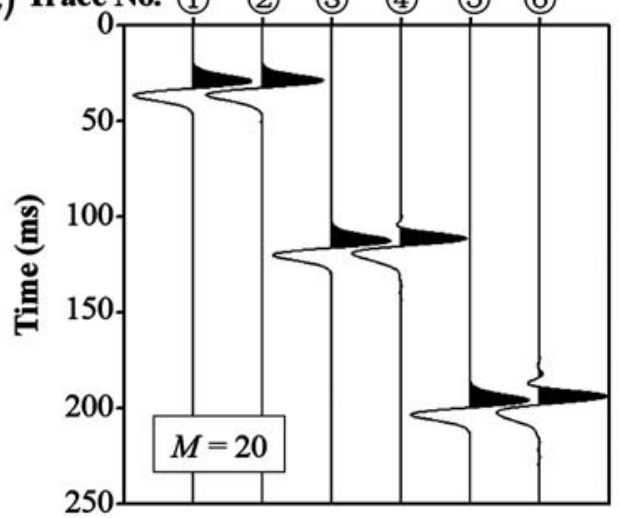

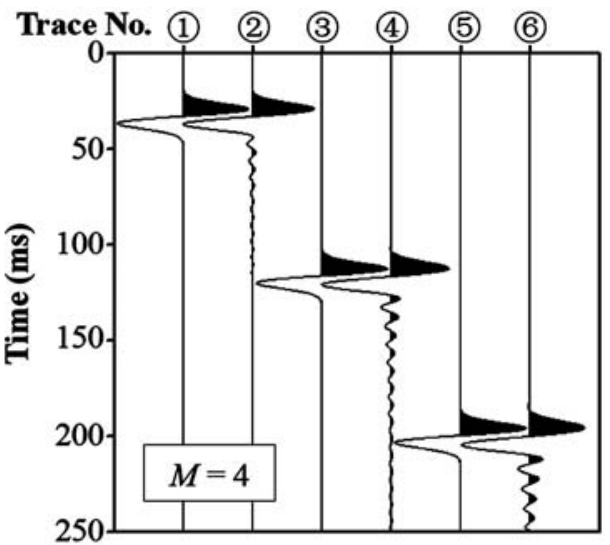
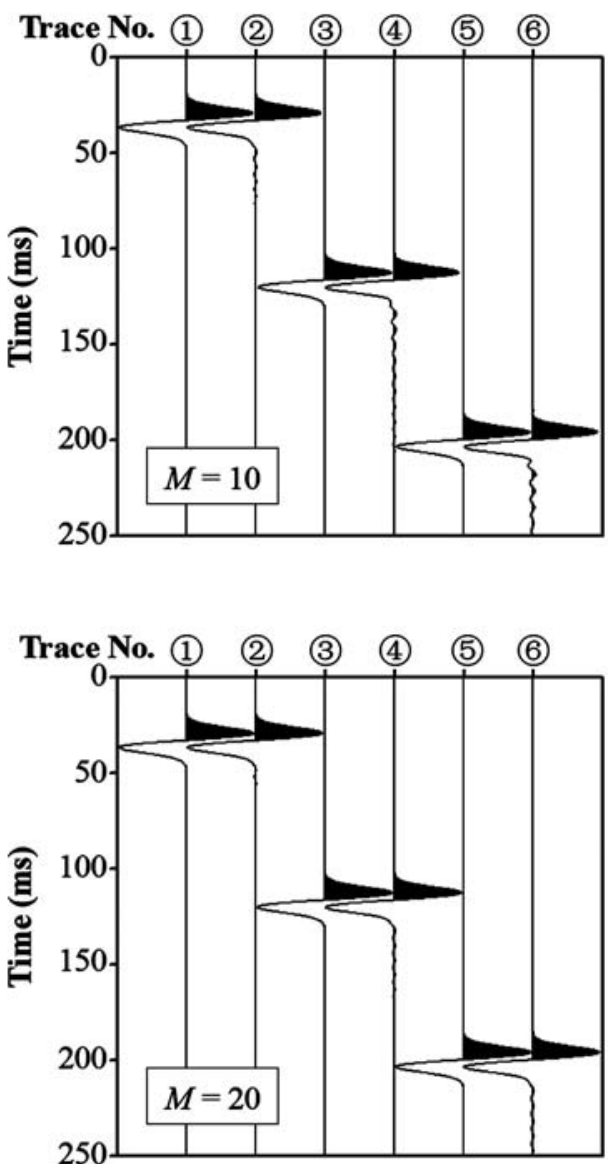

Figure 6. 1D modeling seismograms by (left panels) the conventional methods and (right panels) the new methods for different space point numbers. (1), (3), and (5) are analytic solutions; (2), (4), and (6) are modeling results. Distances between source center and these three receivers are $100 \mathrm{~m}, 350 \mathrm{~m}$, and $600 \mathrm{~m}$, respectively. $\alpha^{2}=1.6, v=3000 \mathrm{~m} / \mathrm{s}, h=10 \mathrm{~m}, \tau=0.001 \mathrm{~s}, M$ values are as shown in each panel.

Because $\delta(\theta)=\delta(\theta+\pi / 2), \delta(\theta)$ is a periodical function with the period of $\pi / 2$. Considering $\delta(\theta)=\delta(\pi / 2-\theta)$, we only calculate $\delta(\theta)$ with the variation of $\theta$ from 0 to $\pi / 4$.

Figure 3 shows the 2D dispersion curves of the conventional and the new methods along three directions for different space point numbers. From the figure, we can see that
- The dispersion generally decreases with the decrease of wavenumber and the increase of $M$.

- The area where $\delta$ nearly equals 1 does not extend with the increase of $M$ for the conventional method, nevertheless the area extends for the new method.

- The accuracy of the new method is generally greater than that of the conventional method for a certain 

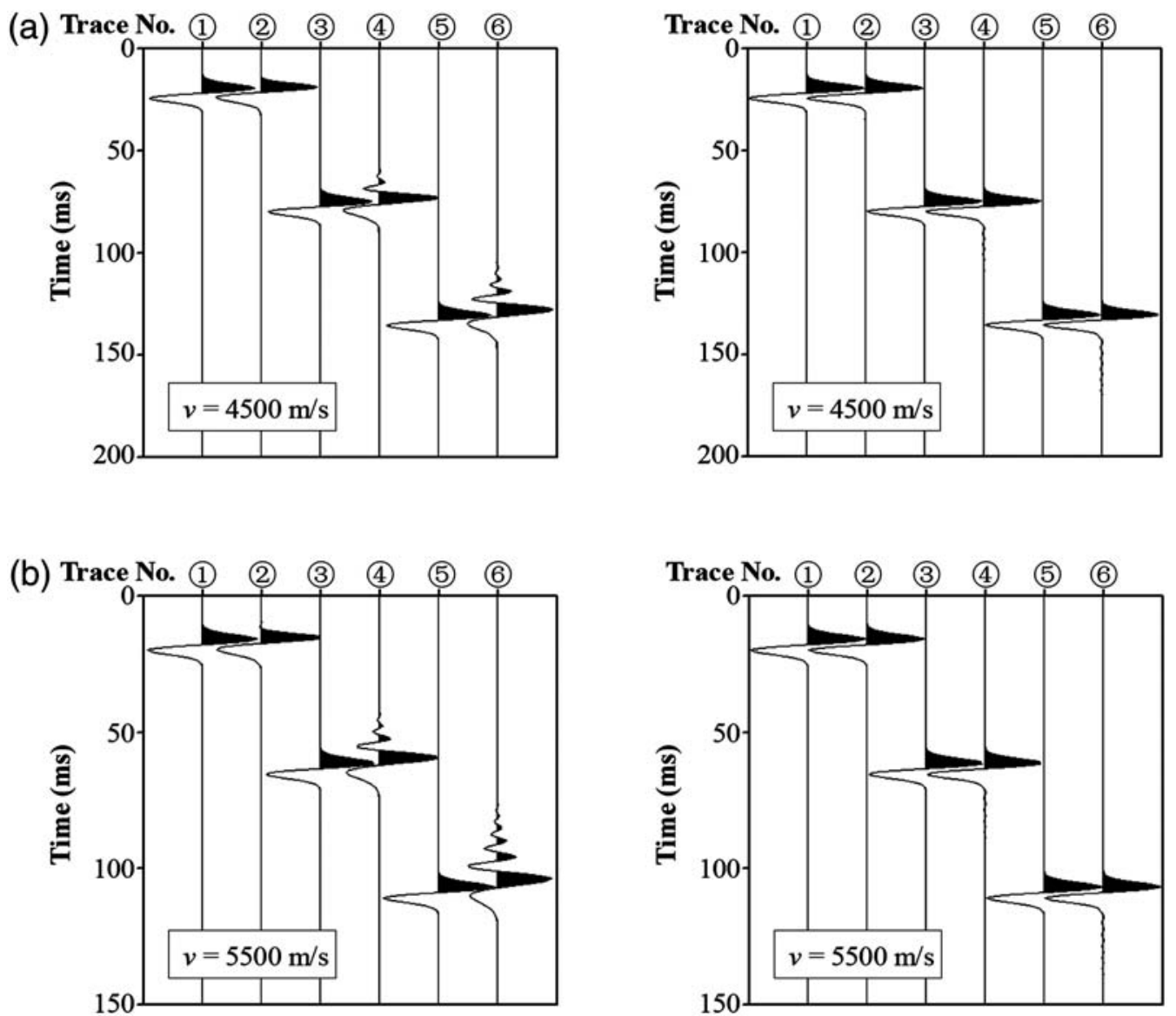

Figure 7. 1D modeling seismograms by (left panels) the conventional methods and (right panels) the new methods for different velocities. (1), (3), and (5) are analytic solutions; (2), (4), and (6) are modeling results. Distances between source center and these three receivers are $100 \mathrm{~m}, 350 \mathrm{~m}$, and $600 \mathrm{~m}$, respectively. $\alpha^{2}=1.6, h=10 \mathrm{~m}, \tau=0.001 \mathrm{~s}, M=20, v$ values are as shown in each panel.

area of $k h$, and the width of this area increases with the increase of $M$.

Figure 4 displays 2D dispersion curves of the conventional and the new methods along five directions when $M=10$, which also demonstrates that the new method has greater accuracy than the conventional method when $k h<2$.

\section{D Dispersion Analysis}

Similarly, 3D dispersion $\delta(\theta, \phi)$ can be defined as

$$
\delta(\theta, \phi)=\frac{v_{\mathrm{FD}}}{v}=\frac{2}{r k h} \sin ^{-1}\left(r \sqrt{q_{3}}\right),
$$

where

$$
\begin{aligned}
q_{3}= & \left(\sum_{m=1}^{M} a_{m} \sin [(m-0.5) k h \cos \theta \cos \phi]\right)^{2} \\
& +\left(\sum_{m=1}^{M} a_{m} \sin [(m-0.5) k h \cos \theta \sin \phi]\right)^{2} \\
& +\left(\sum_{m=1}^{M} a_{m} \sin [(m-0.5) k h \sin \theta]\right)^{2}
\end{aligned}
$$

\section{Stability Analysis}

Stability condition for $n$-dimensional scalar wave equation modeling by the FD method can be expressed as

$$
r \leq s
$$

(see Appendix C), where $s$ is a stability factor,

$$
s=\frac{1}{\sqrt{n}}\left(\sum_{m=1}^{M}\left|a_{m}\right|\right)^{-1} .
$$

\section{D Stability Analysis}

The new method is always stable when $r \leq 1$ because new SFD coefficients are designed for the given $M$ and $r$ (Liu and Sen, 2009c).

\section{D Stability Analysis}

Figure 5a shows the variation of stability factor $s$ with $M$ and $r$ for the new method. The new method is unstable for large values of $r$. The maximum value of $s$ satisfying equation (41) is calculated and shown in Figure 5b with $r$ for the 
(a)

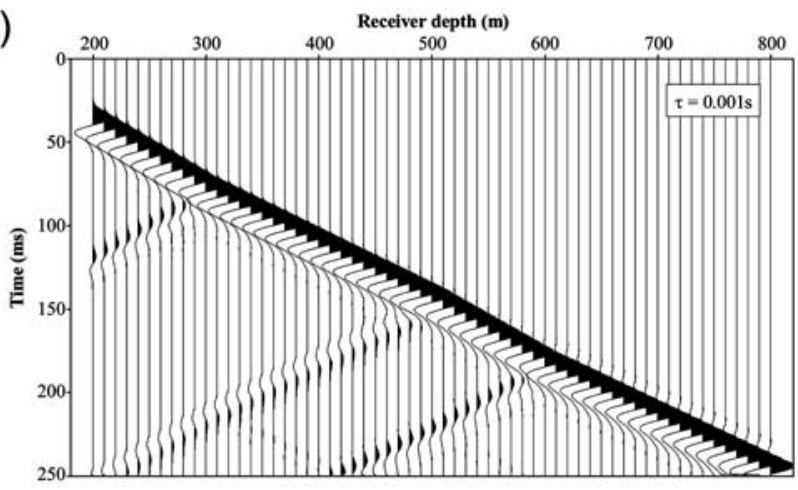

(b)

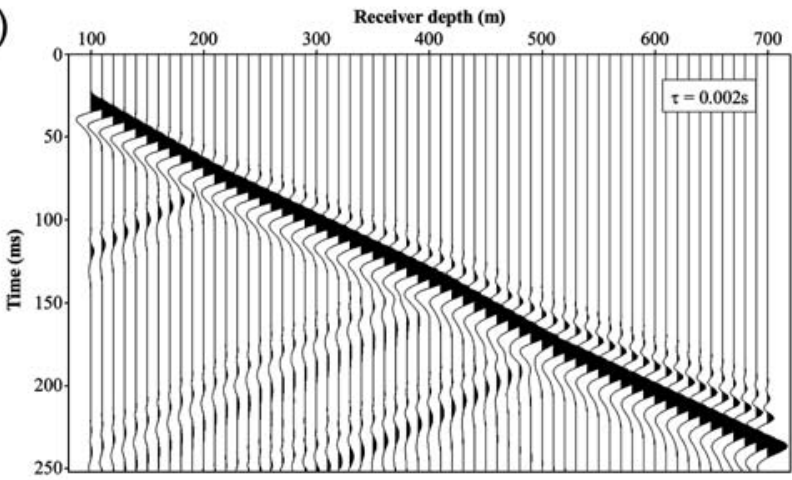

(c)

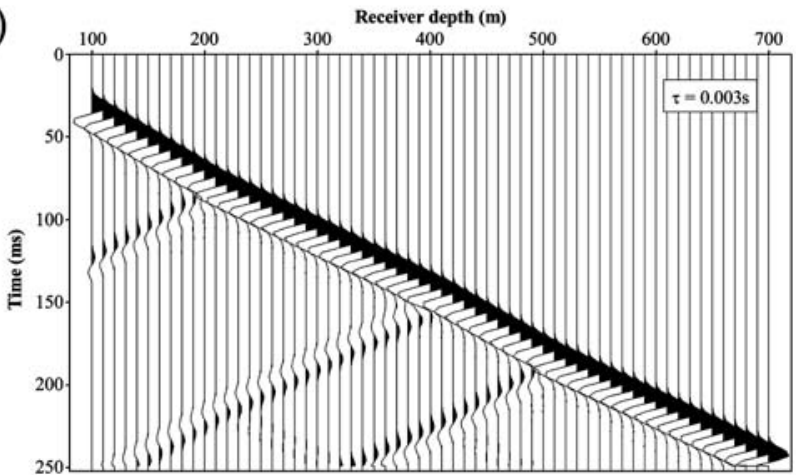

Figure 8. 1D modeling seismograms for an inhomogeneous model by (a,b) the conventional and (c) the new methods. The model has four layers, for which the velocities and densities are $\left(2500 \mathrm{~m} / \mathrm{s}, 2000 \mathrm{~kg} / \mathrm{m}^{3}\right),\left(3000 \mathrm{~m} / \mathrm{s}, 2200 \mathrm{~kg} / \mathrm{m}^{3}\right),(2600 \mathrm{~m} / \mathrm{s}$, $\left.2000 \mathrm{~kg} / \mathrm{m}^{3}\right)$ and $\left(3100 \mathrm{~m} / \mathrm{s}, 2300 \mathrm{~kg} / \mathrm{m}^{3}\right)$, respectively, from shallow to deep. Three interface depths are $300 \mathrm{~m}, 500 \mathrm{~m}$, and $600 \mathrm{~m}$; source depth is $100 \mathrm{~m} . \alpha^{2}=1.6, h=10 \mathrm{~m}, M=10$.

conventional method. The figure demonstrates that the new method can adopt a larger $r$ than the conventional method when $M>1$.

\section{Modeling Examples}

\section{D Modeling}

The initial conditions used in the following 1D numerical modeling are (a)
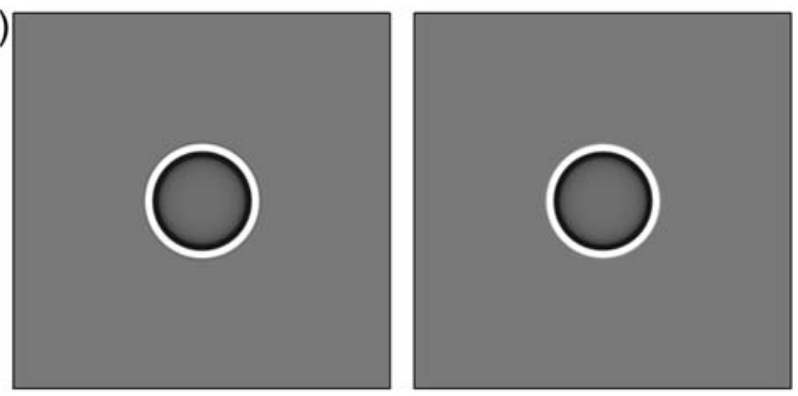

(b)
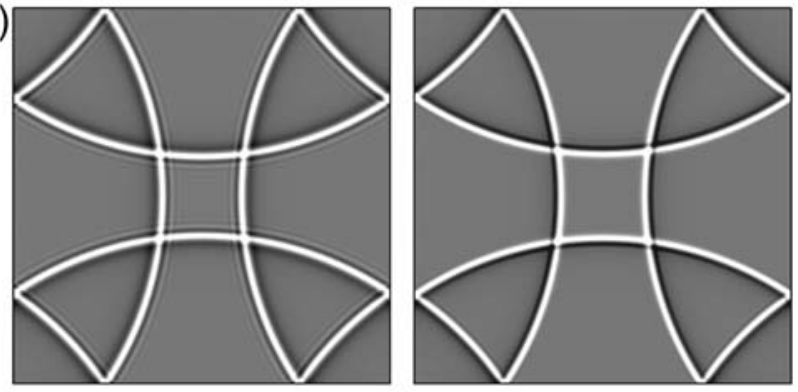

(c)
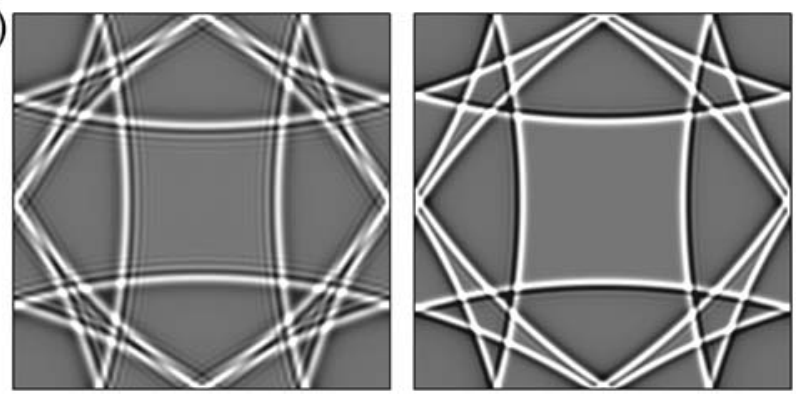

Figure 9. 2D modeling snapshots for a homogeneous model by (left panels) the conventional methods and (right panels) the new methods. Time of the snapshots are (a) $0.10 \mathrm{~s}$, (b) $0.6 \mathrm{~s}$, and (c) $1.2 \mathrm{~s}$. The model is homogeneous, $v=3000 \mathrm{~m} / \mathrm{s}$. The model size is $2000 \mathrm{~m} \times 2000 \mathrm{~m} . \tau=0.001 \mathrm{~s}, h=10 \mathrm{~m}, M=10$. The source is located in the model center. A one-period sine function with $50 \mathrm{~Hz}$ frequency is used to generate vibration.

$$
\left.p(x, t)\right|_{t=0}=\left(x-x_{0}\right) e^{-\frac{a^{2}}{4 h^{2}}\left(x-x_{0}\right)^{2}}
$$

and

$$
\left.\frac{\partial p(x, t)}{\partial t}\right|_{t=0}=0
$$

where $x_{0}$ is the location of the source center, $\alpha^{2}$ is an attenuation coefficient.

Both the conventional and the new SFD methods are used to simulate scalar wave propagation in 1D homogeneous media. Figure 6 shows the seismograms by the conventional and the new methods for different space point numbers. The figure demonstrates that the accuracy increases with the increase of space point number. By comparing with the analytic solutions, we observe that the modeling results from the new method have less dispersion and that the waveforms retain their shapes better than in the conventional 
(a)

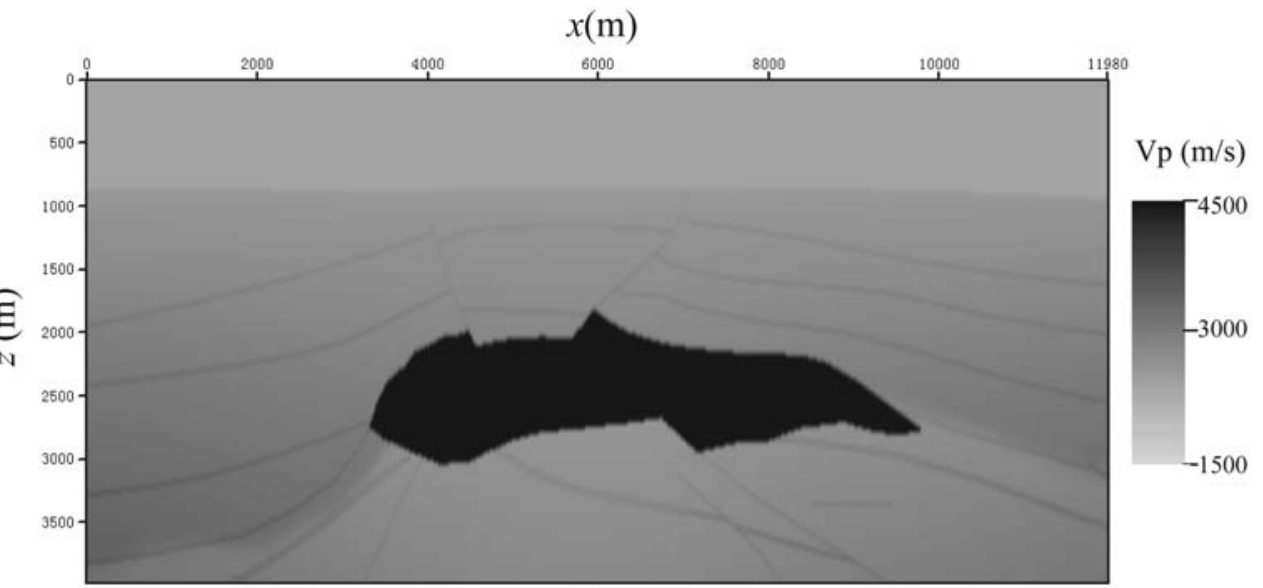

(b)

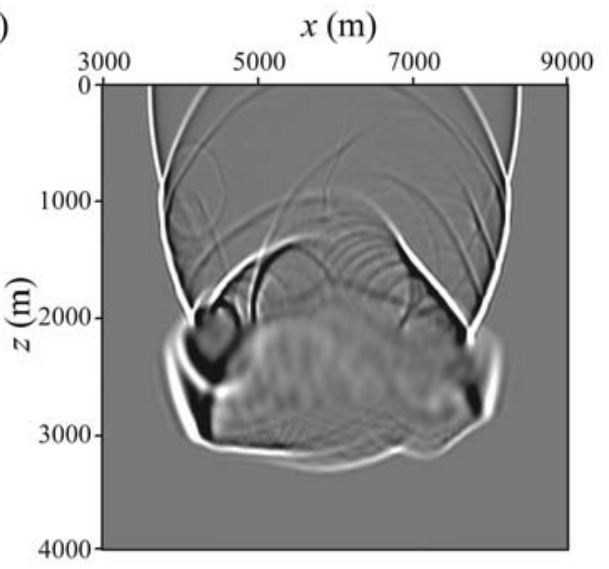

(d)

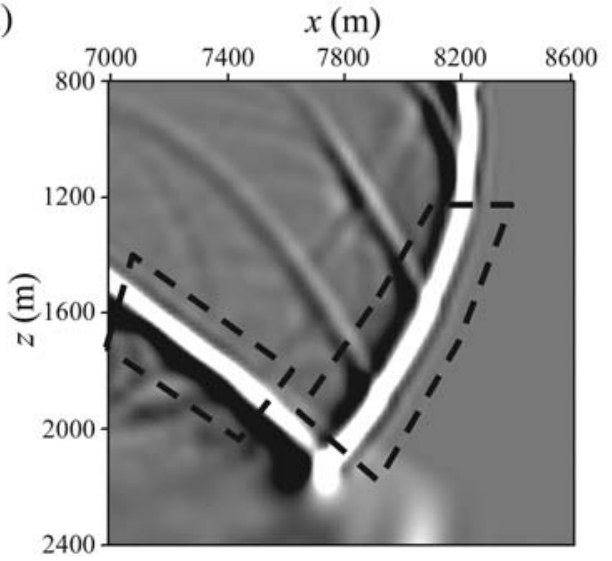

(c)

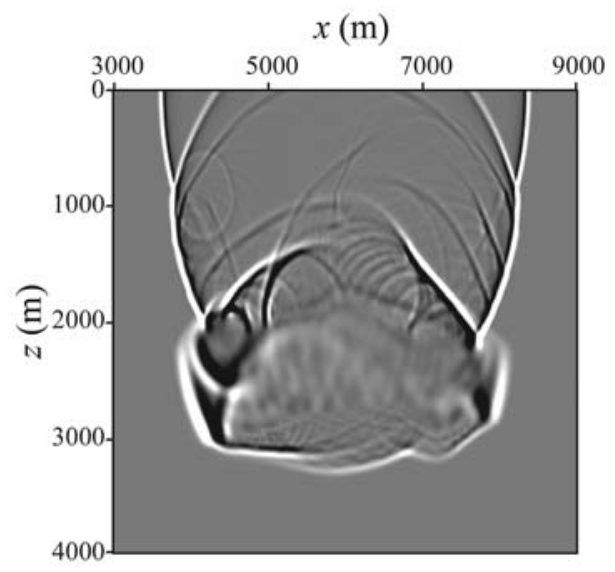

(e)

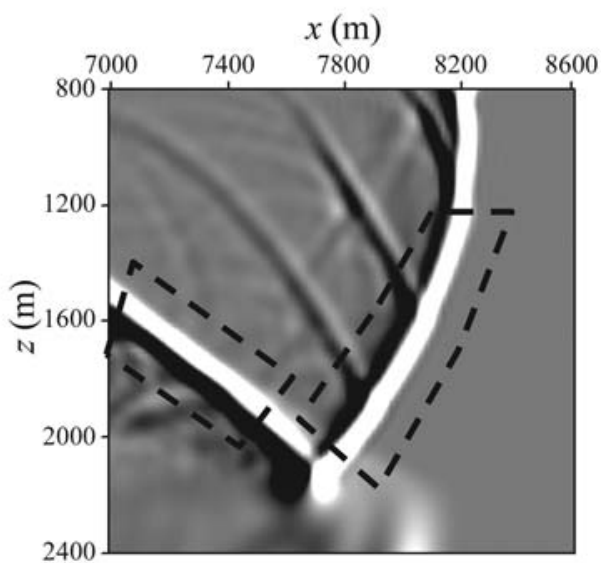

Figure 10. 2D scalar wave modeling snapshots at $1.6 \mathrm{~s}$ and seismograms for (a) the SEG/EAGE salt model of $P$-wave velocity by $(b, f)$ the conventional method and (c, g) the new method. Panels (d) and (e) are zoom images of panels (b) and (c), respectively; and panels (h) and (i) are zoom images of panels (f) and (g), respectively. $h=20 \mathrm{~m}, M=20, \tau=0.002$ s. The source is located at $(6000 \mathrm{~m}, 20 \mathrm{~m})$. A one-period sine function with $20-\mathrm{Hz}$ frequency is used as the source wavelet. 
(f)

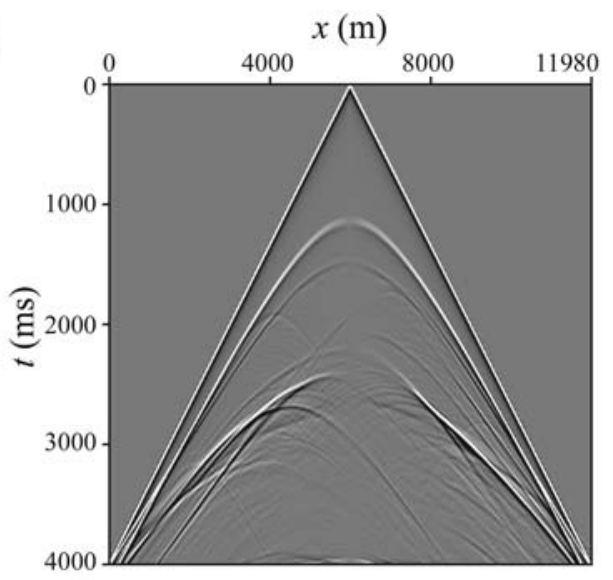

(h)

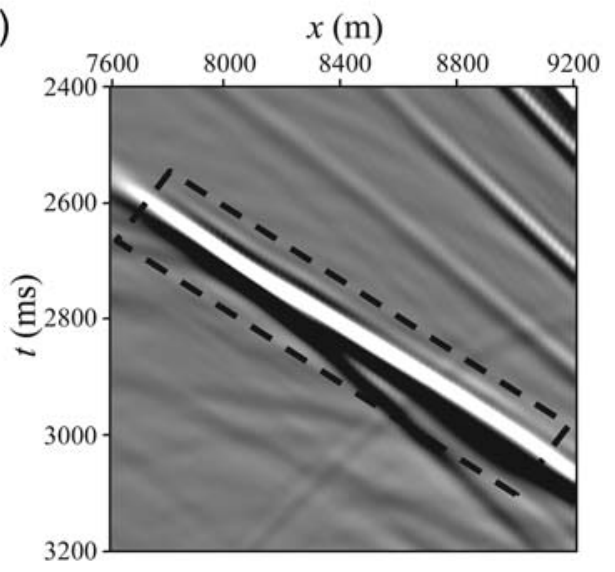

(g)

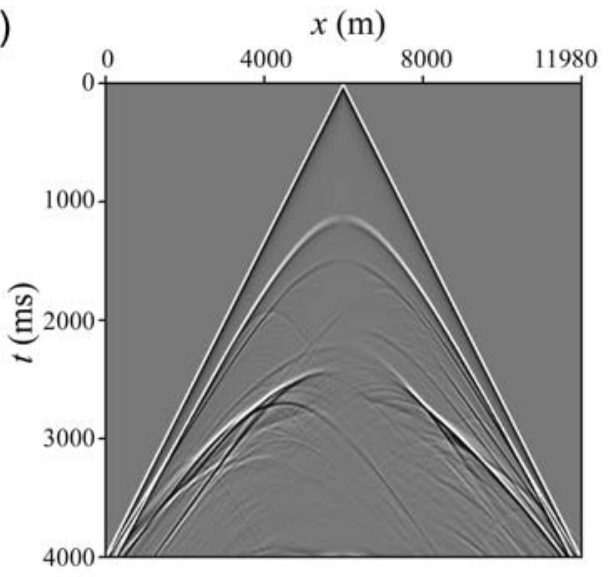

(i)

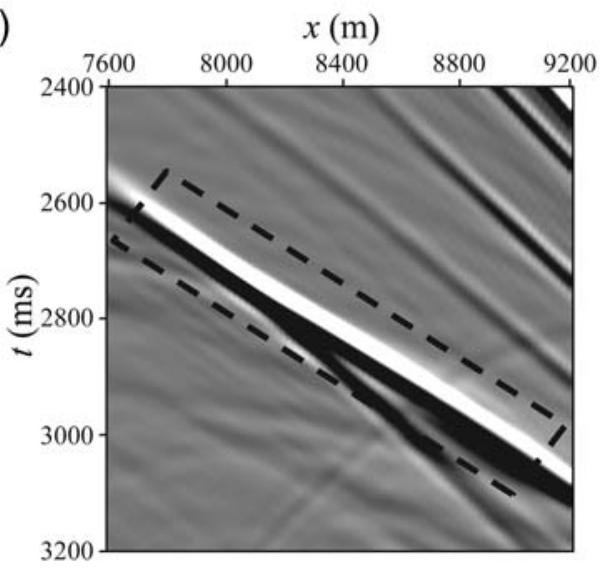

Figure 10. Continued.

method. Figure 7 shows the seismograms for different velocities. The variation of the velocity affects the results from the conventional method more than those from the new method.

The conventional and the new methods are used to perform the numerical modeling for an inhomogeneous model. The records obtained by the numerical modeling are shown in Figure 8. Down-going and up-going waves can be clearly seen in this figure. The conventional method has larger dispersion because the waveform in the record changes significantly with the increase of receiver depth. Because the waveform in the records retains its shape very well for the different receiver depths, the new method is found to have greater accuracy and it effectively suppresses the dispersion.

\section{D Modeling}

The numerical modeling by the conventional and the new methods is performed for a 2D homogeneous model and a heterogeneous model under the same discretization.

Figure 9 displays the snapshots for the homogeneous model. The waveform computed by the new method retains its shape better than the conventional method, which demonstrates that the new method has greater accuracy and smaller dispersion. Figure 10 shows the snapshots and seismograms for a so-called 2D Society of Exploration Geophysicists/ European Association of Geoscientists and Engineers (SEG/EAGE) salt model. Grid dispersion effects can be seen in the result from the conventional method; however, the new method has less dispersion. The modeling results demonstrate that the new 2D method has greater accuracy and less dispersion than the conventional method.

\section{Discussion}

In this section, we discuss the time-space domain dispersion-relation-based spatial SFD scheme for elastic wave equations. Appendix D derives time-space domain dispersion-relation equations (D38) and (D39) by SFD modeling for $2 \mathrm{D}$ elastic wave equations. These equations have the same form as those in equation (20). Using the method presented in the Time-Space Domain Dispersion-Relation-Based Spatial SFD Stencils section, we can obtain the SFD coefficients. 
(a)

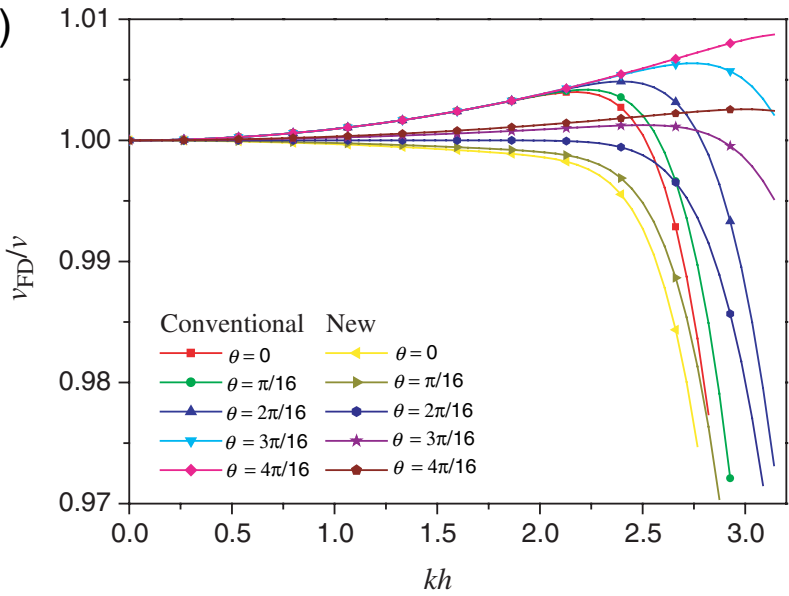

(b)

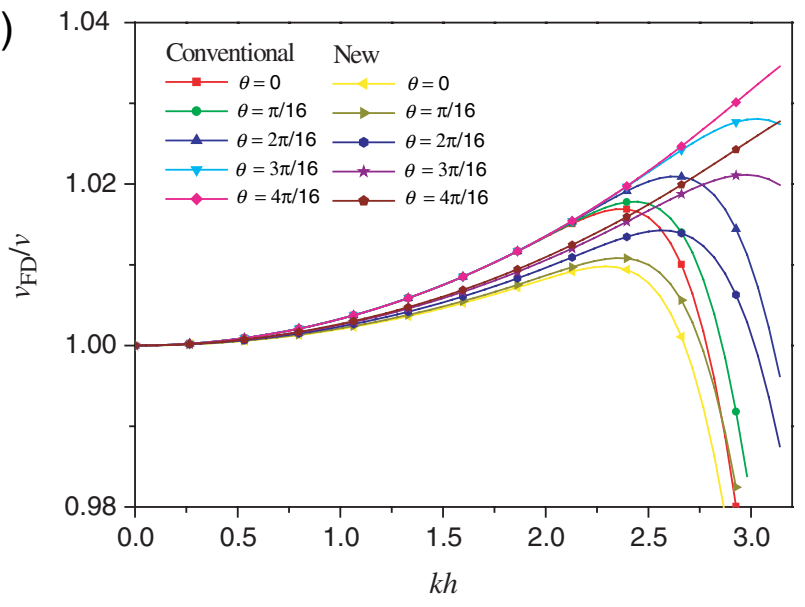

Figure 11. (a) $S$-wave and (b) $P$-wave dispersion curves of the conventional and the new methods for $2 \mathrm{D}$ elastic wave equations. $v_{P}=2800 \mathrm{~m} / \mathrm{s}, \quad v_{S}=1500 \mathrm{~m} / \mathrm{s}, \quad \tau=0.002 \mathrm{~s}, \quad h=20 \mathrm{~m}$, $M=14$. SFD coefficients of new method are determined by $S$ wave velocity. The color version of this figure is available only in the electronic edition.

Note that two velocities, $v_{p}$ and $v_{s}$, exist in each grid in elastic media. However we use only one velocity to design the SFD coefficients. Because the wavenumber of the $S$ wave is less than that of the $P$ wave in each grid, we should use $v_{s}$ to determine the SFD coefficients. However, $v_{P}$ should be used if the medium is fluid.

To demonstrate how the SFD stencil determined by $v_{S}$ works on the $S$ wave and $P$ wave, we calculate 2D $P$-wave and $S$-wave dispersion curves using the conventional and the new methods for various parameters (Figure 11). From this figure, we observe that the accuracy of the new stencil is greater than that of the conventional stencil when $k h<2$. The stencil works on $S$ waves better than on $P$ waves. However, in the same medium, $v_{S}$ is less than $v_{P}$ and the wavenumber of the $P$ wave is less than that of the $S$ wave, so actually the difference of accuracy between $S$-wave dispersion and $P$-wave dispersion is smaller than it looks in this figure. It can be concluded that the SFD stencil determined by $v_{S}$ can work on both the $S$ wave and the $P$ wave and is better than that of the conventional method for a certain area of $k h$.

\section{Conclusions}

We have developed new 2D and 3D spatial SFD stencils based on time-space domain dispersion relations for scalar wave equations where the SFD coefficients are determined by the space point number and the Courant number. The new stencils have greater accuracy than the conventional one under the same discretization. Also, the new stencils can adopt a larger time step. Dispersion analysis and numerical modeling results demonstrate that the new stencils have greater accuracy and can effectively suppress the dispersion and retain the waveform.

\section{Data and Resources}

No data were used in this paper.

\section{Acknowledgments}

This research is partially supported by the National Natural Science Foundation of China under contract 41074100 and the Important National Science \& Technology Specific Project of China under contract 2008ZX05024-001.

\section{References}

Abokhodair, A. A. (2009). Complex differentiation tools for geophysical inversion, Geophysics 74, H1-H11.

Aoi, S., and H. Fujiwara (1999). 3-D finite-difference method using discontinuous grids, Bull. Seismol. Soc. Am. 89, 918-930.

Bansal, R., and M. K. Sen (2008). Finite-difference modelling of $S$-wave splitting in anisotropic media, Geophys. Prospect. 56, 293-312.

Bohlen, T. (2002). Parallel 3-D viscoelastic finite-difference seismic modeling, Comput. Geosci. 28, 887-899.

Claerbout, J. F. (1985). Imaging the Earth's Interior, Blackwell Scientific Publications, Inc., Palo Alto, California, 47-49.

Crase, E. (1990). High-order (space and time) finite-difference modeling of the elastic wave equation, 60th Annual International Meeting, Society of Exploration Geophysicists (SEG), 23-27 September 1990, Expanded Abstracts, 987-991.

Dablain, M. A. (1986). The application of high-order differencing to the scalar wave equation, Geophysics 51, 54-66.

De Basabe, J. D., and M. K. Sen (2007). Grid dispersion and stability criteria of some common finite-element methods for acoustic and elastic wave equations, Geophysics 72, T81-T95.

De Basabe, J. D., and M. K. Sen (2009). New developments in finite element methods of seismic modeling, The Leading Edge 28, 562-567.

De Basabe, J. D., M. K. Sen, and M. F. Wheeler (2008). The interior penalty discontinuous Galerkin method for elastic wave propagation: grid dispersion, Geophys. J. Int. 175, 83-93.

Emerman, S., W. Schmidt, and R. Stephen (1982). An implicit finitedifference formulation of the elastic wave equation, Geophysics 47, $1521-1526$.

Etgen, J. T., and M. J. O’Brien (2007). Computational methods for largescale 3D acoustic finite-difference modeling: a tutorial, Geophysics 72, SM223-SM230.

Finkelstein, B., and R. Kastner (2007). Finite difference time domain dispersion reduction schemes, J. Comput. Phys. 221, 422-438.

Finkelstein, B., and R. Kastner (2008). A comprehensive new methodology for formulating FDTD schemes with controlled order of accuracy and dispersion, IEEE Trans. Antenn. Propag. 56, 3516-3525.

Fornberg, B. (1987). The pseudospectral method-Comparisons with finite differences for the elastic wave equation, Geophysics 52, 483-501. 
Geller, R. J., and N. Takeuchi (1998). Optimally accurate second-order timedomain finite difference scheme for the elastic equation of motion: One-dimensional case, Geophys. J. Int. 135, 48-62.

Graves, R. W. (1996). Simulating seismic wave propagation in 3D elastic media using staggered-grid finite differences, Bull. Seismol. Soc. Am. 86, 1091-1106.

Hayashi, K., and D. R. Burns (1999). Variable grid finite-difference modeling including surface topography, 69th Annual International Meeting, SEG, 31 October-5 November 1999, Expanded Abstracts, 523-527.

Hayashi, K., D. R. Burns, and M. N. Toksöz (2001). Discontinuous-grid finite-difference seismic modeling including surface topography, Bull. Seismol. Soc. Am. 91, 1750-1764.

Hestholm, S. (2003). Elastic wave modeling with free surfaces: Stability of long simulations, Geophysics 68, 314-321.

Hestholm, S., and B. Ruud (1998). 3-D finite-difference elastic wave modeling including surface topography, Geophysics 63, 613-622.

Igel, H., P. Mora, and B. Riollet (1995). Anisotropic wave propagation through finite-difference grids, Geophysics 60, 1203-1216.

Käser, M., and M. Dumbser (2006). An arbitrary high-order discontinuous Galerkin method for elastic waves on unstructured meshes-I. The two-dimensional isotropic case with external source terms, Geophys. J. Int. 166, 855-877.

Kelly, K. R., R. Ward, W. S. Treitel, and R. M. Alford (1976). Synthetic seismograms: A finite-difference approach, Geophysics 41, 2-27.

Kindelan, M., A. Kamel, and P. Sguazzero (1990). On the construction and efficiency of staggered numerical differentiators for the wave equation, Geophysics 55, 107-110.

Komatitsch, D., and J. Vilotte (1998). The spectral element method: An efficient tool to simulate the seismic response of 2D and 3D geological structures, Bull. Seismol. Soc. Am. 88, 368-392.

Kosloff, D., and E. Baysal (1982). Forward modeling by a Fourier method, Geophysics 47, 1402-1412.

Kosloff, D., R. Pestana, and H. Tal-Ezer (2008). Numerical solution of the constant density acoustic wave equation by implicit spatial derivative operators, 78th Annual International Meeting, SEG, 13-18 September 1998, Expanded Abstracts, 2057-2061.

Krüger, O. S., E. H. Saenger, and S. Shapiro (2005). Scattering and diffraction by a single crack: An accuracy analysis of the rotated staggered grid, Geophys. J. Int. 162, 25-31.

Liu, Y., and C. C. Li (2000). Study of elastic wave propagation in two-phase anisotropic media by numerical modeling of pseudospectral method, Acta Seismol. Sinica 13, 143-150.

Liu, Y., and M. K. Sen (2009a). A practical implicit finite-difference method: Examples from seismic modeling, J. Geophys. Eng. 6, 231-249.

Liu, Y., and M. K. Sen (2009b). An implicit staggered-grid finite-difference method for seismic modeling, Geophys. J. Int. 179, 459-474.

Liu, Y., and M. K. Sen (2009c). A new time-space domain high-order finitedifference method for the acoustic wave equation, J. Comput. Phys. 228, 8779-8806.

Liu, Y., and M. K. Sen (2009d). Numerical modeling of wave equation by a truncated high-order finite-difference method, Earthq. Sci. 22, 205-213.

Liu, Y., and M. K. Sen (2010). Acoustic VTI modeling with a time-space domain dispersion-relation-based finite-difference scheme, Geophysics 75, A11-A17.

Liu, Y., and X. C. Wei (2005). A stability criterion of elastic wave modeling by Fourier method, J. Geophys. Eng. 2, 153-157.

Liu, Y., and X. C. Wei (2008). Finite-difference numerical modeling with even-order accuracy in two-phase anisotropic media, Appl. Geophys. $\mathbf{5}, 107-114$

Lombard, B., J. Piraux, C. Gélis, and J. Virieux (2008). Free and smooth boundaries in 2D FD schemes transient elastic waves, Geophys. J. Int. 172, 252-261.

Mittet, R. (2002). Free-surface boundary conditions for elastic staggeredgrid modeling schemes, Geophysics 67, 1616-1623.

Moczo, P., J. Kristek, and L. Halada (2000). 3D fourth-order staggered-grid finite-difference schemes: Stability and grid dispersion, Bull. Seismol. Soc. Am. 90, 587-603.
Moczo, P., J. Kristek, V. Vavrycuk, R. Archuleta, and L. Halada (2002). Heterogeneous staggered-grid finite-difference modeling of seismic motion with volume harmonic and arithmetic averaging of elastic moduli and densities, Bull. Seismol. Soc. Am. 92, 3042-3066.

Ohminato, T., and B. A. Chouet (1997). A free-surface boundary condition for including 3D topography in the finite-difference method, Bull. Seismol. Soc. Am. 87, 494-515.

Opršal, I., and J. Zahradník (1999). Elastic finite-difference method for irregular grids, Geophysics, 64, 240-250.

Pei, Z. L. (2004). Numerical modeling using staggered-grid high order finite-difference of elastic wave equation on arbitrary relief surface, Oil Geophys. Prospect. 39, 629-634 (abstract in English).

Pitarka, A. (1999). 3D elastic finite-difference modeling of seismic motion using staggered grids with nonuniform spacing, Bull. Seismol. Soc. Am. 89, 54-68.

Pratt, R. G., C. Shin, and G. J. Hicks (1998). Gauss-Newton and full Newton methods in frequency-space seismic waveform inversion, Geophys. $J$. Int. 133, 341-362.

Ravaut, C., S. Operto, L. Improta, J. Virieux, A. Herrero, and P. Dell'Aversana (2004). Multiscale imaging of complex structures from multifold wide-aperture seismic data by frequency-domain full-waveform tomography: Application to a thrust belt, Geophys. J. Int. 159, 1032-1056.

Reshef, M., D. Kosloff, M. Edwards, and C. Hsiung (1988a). Three-dimension acoustic modeling by the Fourier method, Geophysics 53, 1175-1183.

Reshef, M., D. Kosloff, M. Edwards, and C. Hsiung (1988b). Threedimension elastic modeling by the Fourier method, Geophysics 53, 1184-1193.

Ristow, D., and T. Ruhl (1994). Fourier finite-difference migration, Geophysics 59, 1882-1893.

Rivière, B., and M. Wheeler (2003). Discontinuous finite element methods for acoustic and elastic wave problems, Contemp. Math. 329, 271-282.

Robertsson, J. O. A. (1996). A numerical free-surface condition for elastic/ viscoelastic finite-difference modeling in the presence of topography, Geophysics 61, 1921-1934.

Robertsson, J. O. A., J. Blanch, and W. Symes (1994). Viscoelastic finitedifference modeling, Geophysics 59, 1444-1456.

Rojas, O., S. Day, J. Castillo, and L. A. Dalguer (2008). Modelling of rupture propagation using high-order mimetic finite differences, Geophys. J. Int. 172, 631-650.

Saenger, E. H., and T. Bohlen (2004). Finite-difference modeling of viscoelastic and anisotropic wave propagation using the rotated staggered grid, Geophysics 69, 583-591.

Saenger, E. H., and S. A. Shapiro (2002). Effective velocities in fractured media: A numerical study using the rotated staggered finite difference grid, Geophys. Prospect. 50, 183-194.

Saenger, E. H., N. Gold, and S. A. Shapiro (2000). Modeling the propagation of elastic waves using a modified finite-difference grid, Wave Motion 31, 77-92.

Takeuchi, N., and R. J. Geller (2000). Optimally accurate second order timedomain finite difference scheme for computing synthetic seismograms in 2-D and 3-D media, Phys. Earth Planet. In. 119, 99-131.

Tessmer, E. (2000). Seismic finite-difference modeling with spatially varying time steps, Geophysics 65, 1290-1293.

Virieux, J. (1984). SH-wave propagation in heterogeneous media: Velocitystress finite-difference method, Geophysics 49, 1933-1957.

Virieux, J. (1986). $P-S V$ wave propagation in heterogeneous media: velocity stress finite difference method, Geophysics 51, 889-901.

Vossen, V. R., J. O. A. Robertsson, and C. Chapman (2002). Finite-difference modeling of wave propagation in a fluid-solid configuration, Geophysics 67, 618-624.

Wang, Y., and G. T. Schuster (1996). Finite-difference variable grid scheme for acoustic and elastic wave equation modeling, 66th Annual International Meeting, SEG, 10-15 December 1996, Expanded Abstracts, 674-677.

Yomogida, K., and J. T. Etgen (1993). 3-D wave propagation in the Los Angeles basin for the Whittier-Narrows earthquake, Bull. Seismol. Soc. Am. 83, 1325-1344. 
Zhang, G., Y. Zhang, and H. Zhou (2000). Helical finite-difference schemes for 3-D. depth migration, 70th Annual International Meeting, SEG, 6-11 August 2000, Expanded Abstracts, 862-865.

\section{Appendix A}

\section{Error Function of 1D Scalar Wave Equation Modeling by the Conventional SFDM}

The absolute error can be obtained from equation (5) by using equation (6)

$$
\begin{aligned}
\varepsilon= & \mid \frac{1}{h^{2}} \sum_{m=1}^{M} \sum_{n=1}^{M} a_{m} a_{n}[2 \cos ((m+n-1) k h) \\
& -2 \cos ((m-n) k h)]-\frac{1}{v^{2} \tau^{2}}[-2+2 \cos (\omega \tau)] \mid \\
= & \mid\left[\frac{2}{h} \sum_{m=1}^{M} a_{m} \sin ((m-0.5) k h)\right]^{2} \\
& +\frac{2}{v^{2} \tau^{2}}[-1+\cos (\omega \tau)] \mid .
\end{aligned}
$$

Substituting equation (10) and equation (11) into equation (A1) and using $v=\omega / k$ and $r=v \tau / h$ to simplify it, we have

$$
\begin{aligned}
\varepsilon= & \mid\left[k+\frac{2}{h} \sum_{j=M+1}^{\infty}\left(\sum_{m=1}^{M}(m-0.5)^{2 j-1} a_{m}\right)\right. \\
& \left.\times \frac{(-1)^{j-1}(h k)^{2 j-1}}{(2 j-1) !}\right]^{2}-\frac{\omega^{2}}{v^{2}}+\frac{2}{v^{2} \tau^{2}} \sum_{j=2}^{\infty}(-1)^{j} \frac{(\omega \tau)^{2 j}}{(2 j) !} \mid \\
= & \mid 2 \sum_{j=2}^{\infty} \frac{(-1)^{j} r^{2 j-2} k^{2 j}}{(2 j) !} h^{2 j-2} \\
& +4 \sum_{j=M+1}^{\infty}\left(\sum_{m=1}^{M}(m-0.5)^{2 j-1} a_{m}\right) \frac{(-1)^{j-1} k^{2 j}}{(2 j-1) !} h^{2 j-2} \\
& +4\left[\sum_{j=M+1}^{\infty}\left(\sum_{m=1}^{M}(m-0.5)^{2 j-1} a_{m}\right)\right. \\
& \left.\times \frac{(-1)^{j-1} k^{2 j-1}}{(2 j-1) !} h^{2 j-2}\right]^{2} \mid .
\end{aligned}
$$

\section{Appendix B}

New Spatial SFD Coefficients for 2D Scalar Wave Equation Modeling

According to the new 2D SFD method, spatial SFD coefficients can be expressed as follows when $M=2,3,4$ :

1. When $M=2$,

$$
a_{2}=\xi_{2}, \quad a_{1}=1-3 a_{2} ;
$$

2. When $M=3$,

$$
\begin{aligned}
& a_{3}=\xi_{3}, \quad a_{2}=\xi_{2}-5 a_{3}, \\
& a_{1}=1-3 a_{2}-5 a_{3} ;
\end{aligned}
$$

3. When $M=4$,

$$
\begin{aligned}
& a_{4}=\xi_{4}, \quad a_{3}=\xi_{3}-7 a_{4}, \\
& a_{2}=\xi_{2}-5 a_{3}-14 a_{4}, \\
& a_{1}=1-3 a_{2}-5 a_{3}-7 a_{4}
\end{aligned}
$$

where

$$
\xi_{2}=\frac{r^{2}}{24\left(\cos ^{4} \theta+\sin ^{4} \theta\right)}-\frac{1}{24},
$$

$$
\begin{aligned}
\xi_{3}= & \frac{\left(2 \beta_{1} \beta_{3}+\beta_{2}^{2}\right) r^{4}}{3840 \beta_{1} \beta_{3}\left(\cos ^{6} \theta+\sin ^{6} \theta\right)} \\
& -\frac{\beta_{2}^{2}\left(1+24 \xi_{2}\right)^{2}}{3840 \beta_{1} \beta_{3}}-\frac{1}{1920}-\frac{1}{8} \xi_{2},
\end{aligned}
$$

$$
\begin{aligned}
\xi_{4}= & \frac{\left(\beta_{1} \beta_{4}+\beta_{2} \beta_{3}\right) r^{6}}{322560 \beta_{1} \beta_{4}\left(\cos ^{8} \theta+\sin ^{8} \theta\right)} \\
& -\frac{\beta_{2} \beta_{3}\left(1+24 \xi_{2}\right)\left(1+240 \xi_{2}+1920 \xi_{3}\right)}{322560 \beta_{1} \beta_{4}} \\
& -\frac{1}{322560}-\frac{13 \xi_{2}}{1920}-\frac{5 \xi_{3}}{24} .
\end{aligned}
$$

\section{Appendix $\mathrm{C}$}

Stability Conditions for Scalar Wave Equation Modeling by SFDMs

First, we derive the 1D stability condition. The 1D recursion equation of SFDMs can be obtain from equation (5) as follows

$$
\begin{aligned}
p_{0}^{1}= & 2 p_{0}^{0}+r^{2} \sum_{m=1}^{M} \sum_{n=1}^{M} a_{m} a_{n}\left[\left(p_{m+n-1}^{0}-p_{m-n}^{0}\right)\right. \\
& \left.-\left(p_{-m+n}^{0}-p_{-m-n+1}^{0}\right)\right]-p_{0}^{-1} .
\end{aligned}
$$

Using the conventional eigenvalue method of stability analysis, we let

$$
q_{m}^{0}=p_{m}^{-1}, \quad U_{m}^{0}=\left(p_{m}^{0}, q_{m}^{0}\right)^{T}=\boldsymbol{W}^{0} e^{i k m h} .
$$

According to equation (C1) and equation (C2), we obtain

$$
\boldsymbol{W}^{1}=\boldsymbol{G} \boldsymbol{W}^{0}=\left[\begin{array}{cc}
g & -1 \\
1 & 0
\end{array}\right] \boldsymbol{W}^{0},
$$


where $\boldsymbol{G}$ is a transition matrix,

$$
g=2-4 r^{2}\left(\sum_{m=1}^{M} a_{m} \sin ((m-0.5) k h)\right)^{2} .
$$

When the absolute values of the transition matrix eigenvalues are less than or equal to 1 , the recursion is stable. If $|g| \leq 2$, the roots of the eigenvalue equation $\lambda^{2}-g \lambda+1=0$ will be less than or equal to 1 . Thus, we have the stability condition

$$
r^{2}\left(\sum_{m=1}^{M} a_{m} \sin ((m-0.5) k h)\right)^{2} \leq 1 .
$$

When

$$
r \leq\left(\sum_{m=1}^{M}\left|a_{m}\right|\right)^{-1},
$$

equation (C5) will be satisfied. Therefore, equation (C6) is the $1 \mathrm{D}$ stability condition.

Similarly, we obtain 2D and 3D stability conditions of

$$
r \leq \frac{1}{\sqrt{2}}\left(\sum_{m=1}^{M}\left|a_{m}\right|\right)^{-1}
$$

and

$$
r \leq \frac{1}{\sqrt{3}}\left(\sum_{m=1}^{M}\left|a_{m}\right|\right)^{-1} .
$$

\section{Appendix D}

Time-Space Domain Dispersion-Relation Formulas of the SFD Solution for 2D Elastic Wave Equations

In this appendix, based on the SFD scheme for 2D elastic wave equations, we derive the time-space domain dispersion-relation formulas.

\section{SFD Algorithm for 2D Elastic Wave Equations} are

The elastic wave equations in 2D heterogeneous media

$$
\begin{gathered}
\frac{\partial v_{x}}{\partial t}=b\left(\frac{\partial \tau_{x x}}{\partial x}+\frac{\partial \tau_{x z}}{\partial z}\right), \\
\frac{\partial v_{z}}{\partial t}=b\left(\frac{\partial \tau_{x z}}{\partial x}+\frac{\partial \tau_{z z}}{\partial z}\right), \\
\frac{\partial \tau_{x x}}{\partial t}=(\lambda+2 u) \frac{\partial v_{x}}{\partial x}+\lambda \frac{\partial v_{z}}{\partial z},
\end{gathered}
$$

$$
\frac{\partial \tau_{z z}}{\partial t}=\lambda \frac{\partial v_{x}}{\partial x}+(\lambda+2 u) \frac{\partial v_{z}}{\partial z},
$$

and

$$
\frac{\partial \tau_{z x}}{\partial t}=\mu\left(\frac{\partial v_{z}}{\partial x}+\frac{\partial v_{x}}{\partial z}\right)
$$

(Virieux, 1986). In these equations, $\left(v_{x}, v_{z}\right)$ is the velocity vector, $\left(\tau_{x x}, \tau_{z z}, \tau_{x z}\right)$ is a vector containing three components of stress, $\lambda(x, z)$ and $\mu(x, z)$ are Lamé coefficients, $b(x, z)$ is the inverse of density.

The derivatives of elastic wave equations in the staggered square grids can be discretized as follows (Virieux, 1986)

$$
\frac{\partial v_{x}}{\partial t} \approx \frac{u_{0,0}^{1}-u_{0,0}^{0}}{\tau},
$$

$$
\frac{\partial \tau_{x x}}{\partial x} \approx \frac{1}{h} \sum_{m=1}^{M} a_{m}\left(o_{m-1 / 2,0}^{1 / 2}-o_{-m+1 / 2,0}^{1 / 2}\right),
$$

$$
\frac{\partial \tau_{x z}}{\partial z} \approx \frac{1}{h} \sum_{m=1}^{M} a_{m}\left(p_{0, m-1 / 2}^{1 / 2}-p_{0,-m+1 / 2}^{1 / 2}\right),
$$

$$
\frac{\partial v_{z}}{\partial t} \approx \frac{w_{1 / 2,1 / 2}^{1}-w_{1 / 2,1 / 2}^{0}}{\tau},
$$

$$
\frac{\partial \tau_{x z}}{\partial x} \approx \frac{1}{h} \sum_{m=1}^{M} a_{m}\left(p_{m, 1 / 2}^{1 / 2}-p_{-m+1,1 / 2}^{1 / 2}\right),
$$

$$
\frac{\partial \tau_{z z}}{\partial z} \approx \frac{1}{h} \sum_{m=1}^{M} a_{m}\left(q_{1 / 2, m}^{1 / 2}-q_{1 / 2,-m+1}^{1 / 2}\right),
$$

$$
\frac{\partial \tau_{x x}}{\partial t} \approx \frac{o_{1 / 2,0}^{1 / 2}-o_{1 / 2,0}^{-1 / 2}}{\tau},
$$

$$
\frac{\partial v_{x}}{\partial x} \approx \frac{1}{h} \sum_{m=1}^{M} a_{m}\left(u_{m, 0}^{0}-u_{-m+1,0}^{0}\right),
$$

and

$$
\frac{\partial v_{z}}{\partial z} \approx \frac{1}{h} \sum_{m=1}^{M} a_{m}\left(w_{1 / 2, m-1 / 2}^{0}-w_{1 / 2,-m+1 / 2}^{0}\right),
$$

where $h$ is grid size, $\tau$ is time step, $a_{m}$ are SFD coefficients, 


$$
\begin{gathered}
u_{m, j}^{n}=v_{x}(x+m h, z+j h, t+n \tau), \\
w_{m+1 / 2, j+1 / 2}^{n}=v_{z}(x+(m+1 / 2) h, z \\
+(j+1 / 2) h, t+n \tau), \\
o_{m+1 / 2, j}^{n+1 / 2}=\tau_{x x}(x+(m+1 / 2) h, z \\
+j h, t+(n+1 / 2) \tau), \\
\\
p_{m, j+1 / 2}^{n+1 / 2}=\tau_{x z}(x+m h, z+(j+1 / 2) h, t \\
+(n+1 / 2) \tau),
\end{gathered}
$$

and

$$
\begin{aligned}
q_{m+1 / 2, j}^{n+1 / 2}= & \tau_{z z}(x+(m+1 / 2) h, z \\
& +j h, t+(n+1 / 2) \tau) .
\end{aligned}
$$

\section{D Dispersion Relation}

Using the plane wave theory, we let

$$
\begin{gathered}
u_{m, j}^{n}=A e^{i\left[k_{x}(x+m h)+k_{z}(z+j h)-\omega(t+n \tau)\right]}, \\
w_{m+1 / 2, j+1 / 2}^{n}=C e^{i\left[k_{x}(x+m h+0.5 h)+k_{z}(z+j h+0.5 h)-\omega(t+n \tau)\right]},
\end{gathered}
$$

$$
o_{m+1 / 2, j}^{n+1 / 2}=D e^{i\left[k_{x}(x+m h+0.5 h)+k_{z}(z+j h)-\omega(t+n \tau+0.5 \tau)\right]},
$$

$$
p_{m, j+1 / 2}^{n+1 / 2}=E e^{i\left[k_{x}(x+m h)+k_{z}(z+j h+0.5 h)-\omega(t+n \tau+0.5 \tau)\right]},
$$

and

$$
q_{m+1 / 2, j}^{n+1 / 2}=F e^{i\left[k_{x}(x+m h+0.5 h)+k_{z}(z+j h)-\omega(t+n \tau+0.5 \tau)\right]},
$$

where $\omega$ is the angular frequency, $\left(k_{x}, k_{z}\right)$ is the wavenumber vector, $i=\sqrt{-1}$. Substituting equations (D20)-(D24) into (D1)-(D5) and simplifying them, we obtain

$$
g A \approx b f_{x} D+b f_{z} E,
$$

$$
g C \approx b f_{x} E+b f_{z} F
$$

$$
g D \approx(\lambda+2 \mu) f_{x} A+\lambda f_{z} C,
$$

$$
g F \approx \lambda f_{x} A+(\lambda+2 \mu) f_{z} C
$$

$$
g E \approx \mu f_{z} A+\mu f_{x} C
$$

where,

$$
g=-\sin (\omega \tau / 2)
$$

$$
f_{x}=\frac{\tau}{h} \sum_{m=1}^{M} a_{m} \sin \left((m-1 / 2) k_{x} h\right),
$$

and

$$
f_{z}=\frac{\tau}{h} \sum_{m=1}^{M} a_{m} \sin \left((m-1 / 2) k_{z} h\right)
$$

Eliminating $A, C, D, E$, and $F$ from equations (D25)-(D29), we have

$$
\begin{aligned}
g^{4} & -\left(\left(b(\lambda+2 \mu) f_{x}^{2}+b \mu f_{z}^{2}\right)+\left(b \mu f_{x}^{2}+b(\lambda+2 \mu) f_{z}^{2}\right)\right) g^{2} \\
& +\left(b(\lambda+2 \mu) f_{x}^{2}+b \mu f_{z}^{2}\right)\left(b \mu f_{x}^{2}+b(\lambda+2 \mu) f_{z}^{2}\right) \\
& -b^{2}(\lambda+\mu)^{2} f_{x}^{2} f_{z}^{2} \approx 0 .
\end{aligned}
$$

Solving this equation, we obtain

$$
g^{2} \approx v_{P}^{2}\left(f_{x}^{2}+f_{z}^{2}\right)
$$

or

$$
g^{2} \approx v_{S}^{2}\left(f_{x}^{2}+f_{z}^{2}\right)
$$

where

$$
v_{P}^{2}=b(\lambda+2 \mu)
$$

and

$$
v_{S}^{2}=b \mu
$$

Substituting equations (D30)-(D32) into equation (D34) and equation (D35), we obtain 


$$
\begin{aligned}
& \left(\sum_{m=1}^{M} a_{m} \sin \left((m-0.5) k_{x} h\right)\right)^{2} \\
& \quad+\left(\sum_{m=1}^{M} a_{m} \sin \left((m-0.5) k_{z} h\right)\right)^{2} \\
& \approx r_{P}^{-2} \sin ^{2}(0.5 \omega \tau)
\end{aligned}
$$

or

$$
\begin{aligned}
& \left(\sum_{m=1}^{M} a_{m} \sin \left((m-0.5) k_{x} h\right)\right)^{2} \\
& \quad+\left(\sum_{m=1}^{M} a_{m} \sin \left((m-0.5) k_{z} h\right)\right)^{2} \\
& \approx r_{S}^{-2} \sin ^{2}(0.5 \omega \tau),
\end{aligned}
$$

where

$$
r_{P}=\frac{v_{P} \tau}{h}
$$

$$
r_{S}=\frac{v_{S} \tau}{h} .
$$

Equation (D38) and equation (D39) are time-space domain dispersion-relation formulas by SFD modeling for 2D elastic wave equations.

State Key Laboratory of Petroleum Resource and Prospecting

China University of Petroleum

Beijing 102249, China

wliuyang@vip.sina.com

(Y.L.)

The Institute for Geophysics

John A. and Katherine G. Jackson School of Geosciences

The University of Texas at Austin

10100 Burnet Road, R2200

Austin, Texas 78758, USA

mrinal@ig.utexas.edu (M.K.S.) 